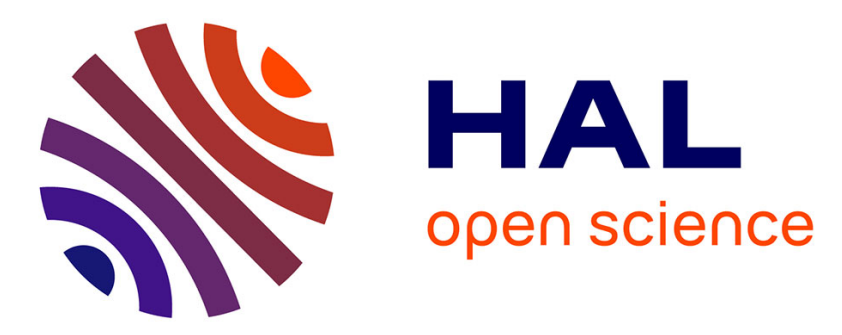

\title{
Impact of coherence decay on wavepacket models for broadband shock-associated noise in supersonic jets
}

Marcus H Wong, Peter Jordan, Damon Honnery, Daniel Edgington-Mitchell

\section{To cite this version:}

Marcus H Wong, Peter Jordan, Damon Honnery, Daniel Edgington-Mitchell. Impact of coherence decay on wavepacket models for broadband shock-associated noise in supersonic jets. Journal of Fluid Mechanics, 2019, 863, pp.969-993. 10.1017/jfm.2018.984 . hal-02351740

\author{
HAL Id: hal-02351740 \\ https://hal.science/hal-02351740
}

Submitted on 6 Nov 2019

HAL is a multi-disciplinary open access archive for the deposit and dissemination of scientific research documents, whether they are published or not. The documents may come from teaching and research institutions in France or abroad, or from public or private research centers.
L'archive ouverte pluridisciplinaire HAL, est destinée au dépôt et à la diffusion de documents scientifiques de niveau recherche, publiés ou non, émanant des établissements d'enseignement et de recherche français ou étrangers, des laboratoires publics ou privés. 


\title{
Impact of coherence decay on wavepacket models for broadband shock-associated noise in supersonic jets
}

\author{
Marcus H. Wong ${ }^{1} \dagger$, Peter Jordan ${ }^{2}$, Damon R. Honnery ${ }^{1}$ and Daniel \\ Edgington-Mitchell ${ }^{1}$
}

\author{
${ }^{1}$ Laboratory for Turbulence Research in Aerospace and Combustion, Department of \\ Mechanical and Aerospace Engineering, Monash University, Melbourne, VIC 3800, Australia \\ ${ }^{2}$ Departement Fluides, Thermique, Combustion, Institut PPRIME, CNRS - Universite de \\ Poitiers - ENSMA, Poitiers, France
}

(Received $\mathrm{xx}$; revised $\mathrm{xx}$; accepted $\mathrm{xx}$ )

Motivated by the success of wavepackets in modelling the noise from subsonic and perfectly-expanded supersonic jets, we apply the wavepacket model to imperfectlyexpanded supersonic jets. Recent studies with subsonic jets have demonstrated the importance of capturing the 'jitter' of wavepackets in order to correctly predict the intensity of far-field sound. Wavepacket jitter may be statistically represented using a two-point coherence function; accurate prediction of noise requires identification of this coherence function. Following the analysis of Cavalieri \& Agarwal (2014), we extend their methodology to model the acoustic sources of broadband shock-associated noise in imperfectly-expanded supersonic jets using cross-spectral densities of the turbulent and shock-cell quantities. The aim is to determine the relationship between wavepacket coherence decay and far-field broadband shock-associated noise, using the model as a vehicle to explore the flow mechanisms at work. Unlike the subsonic case where inclusion of coherence decay amplifies sound pressure level over the whole acoustic spectrum, we find that it does not play such a critical role in determining the peak sound amplitude for shock-cell noise. When higher order shock-cell modes are used to reconstruct the acoustic spectrum at higher frequencies, however, the inclusion of a jittering wavepacket is necessary. These results suggest that the requirement for coherence decay identified in prior BBSAN models is in reality the statistical signature of jittering wavepackets. The results from this modelling approach suggest that nonlinear jittering effects of wavepackets need to be included in dynamic models for broadband shock-associated noise.

\section{Introduction}

When a supersonic jet is operating at off-design conditions, a train of quasi-periodic shock-cells appears. Compared to subsonic or perfectly-expanded supersonic jets, these shock-cells lead to an additional kind of aerodynamic noise. Shock-cell noise is comprised of discrete tones (known as screech) and a broadband component. The generation of screech is due to a self-sustaining feedback loop (Powell 1953) and is believed to be produced between the second and fourth shock-cells (Edgington-Mitchell et al. 2014). The production of broadband shock-associated noise (hereafter BBSAN) follows a similar process (Tam et al. 1986a), but without the resonant feedback loop.

The broadband component is generated by the interaction of downstream-travelling

$\dagger$ Email address for correspondence: marcus.wong@monash.edu 
flow structures with the shock-cells (Harper-Bourne \& Fisher 1973). This interaction produces sound waves that propagate to the far-field, whose maximum intensity is in a direction perpendicular to the jet axis. In the far-field, BBSAN is characterised by a broad spectral peak whose peak frequency is a function of the nozzle pressure ratio (NPR), convection velocity and observer location. The amplitude of BBSAN is dependent on the off-design parameter $\beta=\sqrt{M_{j}^{2}-M_{d}^{2}}$ where $M_{j}$ and $M_{d}$ are the perfectly expanded and design Mach numbers of the jet, respectively.

Models for BBSAN were initially developed based on experimental observation, such as the empirical model of Harper-Bourne \& Fisher (1973) which is based on a phased array of localised sources along the jet centreline. The directivity of BBSAN is proposed to be due to the relative phasing between the sources, where the time delay in acoustic emission between adjacent sources is a function of the convection velocity of the convecting flow structures. While the model is capable of reproducing many of the acoustic far-field features, it incorrectly predicts the occurrence of harmonically related peaks, nor does it account for azimuthal modes other than the axisymmetric mode.

More recently semi-empirical hybrid models have been developed. With the increase in computational power available in recent times, hybrid models are now being used to model BBSAN for different nozzle geometries (Morris \& Miller 2010; Miller \& Morris 2010) with reasonable success. These models use RANS mean-flow solutions to provide input for acoustic-analogy source terms which link flow-field fluctuations to the propagation of sound waves in the far-field (Lighthill 1952). Mixed-scale models (Kalyan \& Karabasov 2017; Tan et al. 2017), using frequency-dependent length scales, have been shown to improve agreement with data over a larger range of frequencies compared to Morris and Miller's original model. The acoustic source models are based on measured bulk two-point turbulence correlations. Such hybrid models are now capable of enabling large parametric studies with fast turnaround times. These semi-empirical models require accurate source descriptions based on turbulence statistics and their construction for a given flow is a non-trivial task. This difficulty motivates the development of simplified reduced-order descriptions of the noise-producing flow features. One such approach involves modelling organised flow structures as hydrodynamic instability waves, or wavepackets as they are now more often called.

The use of wavepackets to represent large-scale coherent structures (Crow \& Champagne 1971) is well documented. Mollo-Christensen (1967) was the first to propose their use to model jet noise and many experiments have been performed that indicate the validity of the approach (Suzuki \& Colonius 2006; Gudmundsson \& Colonius 2011; Cavalieri et al. 2012, 2013) for subsonic jets. The growth, saturation and decay behaviour of wavepackets emulates the downstream evolution of large-scale structures as the jet mean profile spreads. The presence of these acoustically non-compact structures has already been confirmed in the hydrodynamic field of subsonic jets (Suzuki \& Colonius 2006; Gudmundsson \& Colonius 2011; Cavalieri et al. 2013). The reduced-order velocity field educed from PIV measurements matches well with the predictions of linear stability models. These models also predict the axisymmetric superdirectivity and lower-order azimuthal character of jet noise, consistent with measured results (Cavalieri et al. 2012). A comprehensive summary of subsonic jet-noise modelling using wavepackets can be found in the review of Jordan \& Colonius (2013).

Wavepacket models have also been used for supersonic jet noise (Léon \& Brazier 2013; Sinha et al. 2014). The idea of incorporating large-scale structures for modelling BBSAN was first proposed by Tam. In a series of papers by Tam and co-workers (Tam \& Tanna 1982; Tam et al. 1986b; Tam 1987), a dynamic model for BBSAN has been 
proposed wherein noise is produced via weak nonlinear interactions between shock-cells and turbulent structures in the shear layer. The turbulent structures are represented as a superposition of instability waves at different frequencies. As these instability waves convect downstream, they interact with the shock-cells which are represented as a series of stationary waveguide modes. The stochastic description of the model arises from the random fluctuations of the instability waves. Tam's model was found to match well with experimental data though it suffered at upstream angles close to the nozzle exit where non-physical narrow-banded peaks are predicted.

The same observations that motivate dynamic modelling approaches based on stability theory can also be used to construct kinematic models for shock-associated noise in supersonic jets. Unlike dynamic models where the instability modes of the jet and the acoustic field are obtained simultaneously via solution of the linearised flow equations, kinematic sound-source models (Morris \& Miller 2010; Kalyan \& Karabasov 2017) obtain the radiated sound-field via an acoustic analogy. Rather than using the bulk turbulence statistics to construct the source term (Morris \& Miller 2010; Harper-Bourne \& Fisher 1973), however, a wavepacket description consistent with the results of dynamic modelling is here used for the source term (Lele 2005). The wavepacket source term parameters can be educed from carefully planned experiments (Jaunet et al. 2017; Maia et al. 2017), where the modelled fluctuating components are first decomposed into azimuthal modes and in frequency. A kinematic model for broadband shock-associated noise, using a wavepacket representation, was first proposed by Lele (2005). Similar to Tam et al. $(1986 a)$, Lele hypothesised that the sources are associated with the nonlinear interaction of instability waves with the stationary shock-cell modes, represented as a sum of standing waves (Pack 1950). The suitability of using wavepackets to model sound sources in shockcontaining flows was confirmed by the LDV measurements of Savarese et al. (2013).

An important element for wavepacket modelling in subsonic jets is the two-point coherence of the associated azimuthal modes. Wavepacket fluctuations in a jet will exhibit coherence decay with distance due to the action of turbulence. For subsonic jets, its neglect can lead to discrepancies in the far-field of several orders of magnitude (Baqui et al. 2013; Suzuki 2013; Breakey et al. 2013; Jordan et al. 2014; Zhang et al. 2014). By including this phenomena in their kinematic source model, Cavalieri et al. (2011) were able to demonstrate that wavepacket jitter can indeed dramatically increase sound radiation efficiency in subsonic jets. The impact of coherence decay in wavepacket models in predicting far-field noise is discussed in depth by Cavalieri \& Agarwal (2014) who show that a two-point kinematic model with coherence decay is required in subsonic jets in order to match the far-field sound pressure level. By matching the coherence behaviour to turbulent subsonic jets, Baqui et al. (2015) used a linear stability model to show how sensitive far-field predictions are to coherence decay.

For ideally-expanded supersonic flows, however, this jittering behaviour has been shown to be less important, since the main hydrodynamic wavelengths are already acoustically 'matched' (Crighton 1975) and thus able to radiate to the far-field efficiently (Sinha et al. 2014; Cavalieri et al. 2014). This is further supported by the finding of Cheung \& Lele (2009) where nonlinear PSE accurately predicted the far-field acoustics of a supersonic two-dimensional mixing layer but failed in the subsonic case.

It is well recognised that some form of coherence/correlation decay is a controlling parameter in jet noise. This recognition is evident in the significant effort which has been expended to measure the bulk two-point statistics in turbulent jets (Harper-Bourne 2002; Freund 2003; Kerhervé et al. 2004; Jordan \& Gervais 2005; Morris \& Zaman 2010; Pokora \& McGuirk 2015, amongst others). The measurements (typically dominated by the energy-containing eddies), guided the construction of the two-point coherence 
and correlation function in BBSAN-modelling schemes such as Harper-Bourne \& Fisher (1973) and Morris \& Miller (2010) respectively. In shock-containing flows, Lele (2005) demonstrated the effect of coherence decay by introducing it into a localised phasedarray model similar to that of Harper-Bourne \& Fisher. Coherence decay of the bulk turbulence, was found to be effective in controlling the harmonic peaks produced when using a perfectly-coherent source pair. The degree of suppression of the higher-order peaks is dependent on the extent to which the cross-coherence decays between sources. These harmonic peaks were also observed in Tam's dynamic instability wave model.

In the kinematic framework, however, a clear distinction needs to be made between previous BBSAN sound-source models and the proposed wavepacket model. Jaunet et al. (2017) have shown considerable difference between two-point bulk statistics, obtained from point measurements, on one hand, and, on the other, two-point statistics of individual azimuthal modes using dual-plane PIV data of a subsonic turbulent jet. The majority of the fluctuating turbulent energy is contained in scales which correspond to higher-order azimuthal modes. These modes, however, have been shown to be acoustically inefficient (Michalke 1970; Cavalieri et al. 2012). Hence, while two-point BBSAN source models based on bulk-flow statistics (Harper-Bourne \& Fisher 1973; Morris \& Miller 2010) do explicitly include two-point coherence or correlation information, they are not directly equivalent to wavepacket models where only the velocity perturbations of the acoustically efficient lowest-order azimuthal modes are used. Throughout the paper, coherence decay will refer to the two-point coherence behaviour of wavepackets, and not that of bulk turbulence as studied previously such as Harper-Bourne \& Fisher (1973) and Morris \& Miller (2010).

The importance of wavepacket jitter in shock-containing supersonic flows is less clear. Using a model where the turbulence fluctuations are modelled by wavepackets and the shock-cell noise sources as a collection of empirical Gaussian humps, Suzuki (2016) deduced the source parameters from azimuthally decomposed LES near-field pressure data. The acoustic signature of the source was extracted by solving the boundaryvalue problem using the pressure field on a surface surrounding the jet as a boundary for the wave equation. By modelling this acoustic signature in the frequency domain, the coherence decay behaviour was matched for the cases studied. Good agreement at the BBSAN peak frequencies was achieved between the model and data for a range of frequencies. The effect of coherence decay, however, was not discussed.

A further clue to the importance of the aforementioned nonlinear jittering effect, however, can be seen in the results of Ray \& Lele (2007) who extended Tam's dynamic broadband shock-associated noise model. The small-amplitude disturbances were decomposed into azimuthal modes and represented as instability waves. For a cold underexpanded jet, they found good agreement at low frequencies but highlighted that their instability model was unable to capture higher frequencies, which they attributed to 'some combination of nonlinear and non-modal effects'. This suggests that at higher frequencies, the nonlinear effect of wavepacket jitter may play an important role.

In order to develop accurate dynamic models, it is crucial to determine whether coherence decay is important in a given flow. Using kinematic models to 'test' the impact of coherence decay can provide valuable information regarding the forcing term that is required in the dynamic modelling framework (Towne et al. 2015).

In this paper, encouraged by the results of Ray \& Lele (2007) and Suzuki (2016), we extend the model of Cavalieri \& Agarwal (2014) to the study of broadband shockassociated noise. We propose a two-point kinematic model for BBSAN in order to understand the effect of coherence decay in shock containing flows, where the source terms for both the turbulent and shock-cell component are derived from linearised flow 
equations. The departure point is the single-point wavepacket model (equation 4.4.1 from Lele (2005)) that we modify by replacing the time dependence with a term that models the two-point coherence. The remainder of the paper is structured as follows. The mathematical formulation of the two-point wavepacket model is first presented in $\S 2$. In $\S 3$, we highlight the effect of coherence decay on the far-field sound-radiation properties predicted by the model. By comparing to historical experimental data, we specifically look at the acoustic efficiency and directivity for wavepackets interacting with a single shock-cell mode. The interpretation of sound radiating characteristics of the model is then discussed in $\S 4$. The effect of coherence decay on the more general model of multiple shock-cell modes is presented in $\S 5$ with conclusions and future perspectives provided in $\S 6$.

\section{Mathematical model}

The kinematic wavepacket sound-source model is based on Lighthill's acoustic analogy, where the fluctuating sound pressure, $p$, is given by the inhomogeneous wave equation

$$
\nabla^{2} p-\frac{1}{c_{0}^{2}} \frac{\partial^{2} p}{\partial t^{2}}=S(\boldsymbol{y}, t),
$$

where $\boldsymbol{y}$ are the source coordinates, $c_{0}$ is the ambient speed of sound, $t$ is time and $S(\boldsymbol{y}, t)$ is the source term expressed as the double divergence of Lighthill's stress tensor along the jet axis. The Helmholtz equation can be obtained via a Fourier transform of equation (2.1) to arrive at

$$
\nabla^{2} p+k^{2} p=S(\boldsymbol{y}, \omega)
$$

where $k=\omega / c_{0}$.

Using a free-field Green's function $G(\boldsymbol{x}, \boldsymbol{y}, \omega)$, the integral solution to the Helmholtz equation (2.2) is,

$$
p(\boldsymbol{x}, \omega)=\int_{V} S(\boldsymbol{y}, \omega) G(\boldsymbol{x}, \boldsymbol{y}, \omega) d \boldsymbol{y},
$$

where the integration is carried out over the region $V$ where $S \neq 0$ and $\boldsymbol{x}$ are the observer coordinates.

As proposed by Tam (1990) and Lele (2005), the full three-dimensional source term for BBSAN can be represented as a one-dimensional multiplicative combination of the shock-cell $u_{s}$ and turbulent $u_{t}$ velocity fluctuations

$$
S(\boldsymbol{y}, t) \simeq \hat{S}(y, t)=u_{s}(y) u_{t}(y, t),
$$

where $\hat{S}(y, t)$ is a line-source model (azimuthal and radial dependence are not considered). The coordinate vector $\boldsymbol{y}$ is now replaced by the axial position coordinate $y$. This modelling of acoustic sources along a line thus only accounts for axisymmetric wavepacket fluctuations.

One approach to model the disturbances due to the quasi-periodic train of shockcells is to regard the jet mixing layer as a waveguide (Prandtl 1904; Pack 1950). By approximating the mixing layer of the jet as a vortex sheet, the disturbances due to the shock-cells can be decomposed into a set of spatially periodic functions. Each of these periodic functions can be thought of as a waveguide mode of the jet. In one dimension, the 
velocity fluctuations related to the shock-cell waveguide modes along the axial direction, $u_{s}(y)$, is represented as (Prandtl 1904; Pack 1950)

$$
u_{s}(y)=U_{s} \sum_{n} c_{s_{n}} \frac{1}{2}\left\{e^{\mathrm{i} k_{s_{n}} y}+e^{-\mathrm{i} k_{s_{n}} y}\right\} .
$$

The shock-cell waveguide modes are described by the wavenumbers $k_{s_{n}}$ and the amplitude terms $c_{s_{n}}$ where we adopt the expression from Prandtl \& Pack's vortex sheet model,

$$
\begin{array}{cc}
k_{s_{n}}=\frac{2 \sigma_{n}}{D_{j}\left(M_{j}^{2}-M_{d}^{2}\right)^{1 / 2}}, & n=1,2,3 \ldots, \\
c_{s_{n}} \quad=\frac{2 \triangle p}{\sigma_{n} p_{\infty}}, & n=1,2,3 \ldots,
\end{array}
$$

where $\sigma_{n}$ is the $n^{\text {th }}$ zero-crossing of the zeroth-order Bessel function, $D_{j}$ and $M_{j}$ are respectively, the ideally-expanded diameter and Mach number of the jet. $M_{d}$ is the design Mach number which is equal to unity for a convergent nozzle. The amplitude term $c_{s_{n}}$ is the ratio between the pressure imbalance $\triangle p$ at the throat of the nozzle and the ambient pressure $p_{\infty}$. The amplitude decay of the shock modes over axial distance as seen in experimental measurements, however, is not calculated or accounted for. The overall scaling amplitude of the shock-cells is represented by $U_{s}$. The complete solution for the vortex sheet shock-cell model can be found in Pack (1950).

To model $u_{t}$, Lele (2005) used a wavepacket whose amplitude is modulated in both space and time. The wavepacket, at a given axial position $y$, is defined by its envelope length scale $L$, hydrodynamic wavenumber $k_{h}$ and frequency $\omega$; the two latter quantities being related by the convection velocity $k_{h}=\omega / U_{c}$. The explicit single-point form of $u_{t}$ with amplitude $U_{t}$ is,

$$
u_{t}(y, t)=U_{t} e^{-\left(\frac{y}{L}\right)^{2}} e^{\mathrm{i}\left(k_{h} y-\omega t\right)}
$$

To model the jitter of the wavepackets, Lele introduced a temporal modulation term involving stochastic realisations. The work of Cavalieri \& Agarwal (2014) however, established that coherence decay can provide an alternative statistical representation of jitter. Therefore, rather than including a temporal dependence, we use two-point statistics to model the wavepacket's stochastic behaviour. After taking the Fourier transform of the proposed source model $\hat{S}(y, \omega)$, the source term at a single point $y$ is now given by

$$
\hat{S}(y, \omega)=A(\omega) e^{-\left(\frac{y}{L(\omega)}\right)^{2}}\left\{e^{\mathrm{i} k_{h}(\omega) y}\right\} \sum_{n} c_{s_{n}}\left\{e^{\mathrm{i} k_{s_{n}} y}+e^{-\mathrm{i} k_{s_{n}} y}\right\}
$$

where an implicit factor of $\exp (-i \omega t)$ is assumed and the $U_{s}$ and $U_{t}$ amplitude terms in equation (2.5 and 2.8) have been absorbed into the amplitude term $A(\omega)$.

While equation (2.9) allows direct computation of the fluctuating pressure field, the Fourier transform that would provide the source term $S(\boldsymbol{y}, \omega)$ cannot be evaluated as it involves an integrand that is not square-integrable (Landahl \& Mollo-Christensen 1992; Cavalieri \& Agarwal 2014). Fluctuations in a turbulent jet comprise a stationary random process, and are best described through statistical metrics such as variance, autocorrelation and cross-correlation. In the frequency domain, a particularly rich statistical metric is the cross-spectral density, which is the Fourier transform of the cross-correlation, but which can also be defined as the expected value $\mathcal{E}\left(\hat{u} \hat{u}^{*}\right)$, where $\hat{u}$ is the Fourier transform taken for a given realisation, and $\mathcal{E}$ is the expected-value operator, which is the asymptotic limit of an ensemble average. Using power-spectral densities (PSDs) and cross-spectral 
densities (CSDs), the Fourier transforms of the autocorrelation and cross-correlation functions, respectively, we express the far-field sound pressure level (SPL) as

$$
\left\langle p(\boldsymbol{x}, \omega) p^{*}(\boldsymbol{x}, \omega)\right\rangle \approx \int_{V} \int_{V}\left\langle S\left(y_{1}, \omega\right) S^{*}\left(y_{2}, \omega\right)\right\rangle G\left(\boldsymbol{x}, y_{1}, \omega\right) G^{*}\left(\boldsymbol{x}, y_{2}, \omega\right) d y_{1} d y_{2},
$$

where both PSDs and CSDs are obtained by multiplying by the complex conjugate between position $y_{1}$ and $y_{2}$ and the hats have been dropped for convenience. The freefield Green's function is

$$
G(\boldsymbol{x}, \boldsymbol{y}, \omega)=\frac{1}{4 \pi} \frac{e^{\mathrm{i} k|\boldsymbol{x}-\boldsymbol{y}|}}{|\boldsymbol{x}-\boldsymbol{y}|} .
$$

To obtain the two-point source term with unit coherence at position $y_{1}$, we multiply equation 2.9 by its complex conjugate at position $y_{2}$

$$
\begin{aligned}
S\left(y_{1}, \omega\right) S^{*}\left(y_{2}, \omega\right)= & A^{2}(\omega) e^{-\left(\frac{y_{1}}{L(\omega)}\right)^{2}} e^{-\left(\frac{y_{2}}{L(\omega)}\right)^{2}}\left\{e^{\mathrm{i} k_{h}(\omega)\left(y_{1}-y_{2}\right)}\right\} \times \\
& \sum_{n} c_{s_{n}}\left\{e^{\mathrm{i} k_{s_{n}} y_{1}}+e^{-\mathrm{i} k_{s_{n}} y_{1}}\right\} \sum_{m} c_{s_{m}}\left\{e^{\mathrm{i} k_{s_{m}} y_{2}}+e^{-\mathrm{i} k_{s_{m}} y_{2}}\right\}
\end{aligned}
$$

We now define the coherence function between points $y_{1}$ and $y_{2}$ as

$$
\gamma^{2}\left(y_{1}, y_{2}, \omega\right)=\frac{\left|\left\langle S\left(y_{1}, \omega\right) S^{*}\left(y_{2}, \omega\right)\right\rangle\right|^{2}}{\left\langle\left|S\left(y_{1}, \omega\right)\right|^{2}\right\rangle\left\langle\left|S\left(y_{2}, \omega\right)\right|^{2}\right\rangle}
$$

modelled as a Gaussian function following Cavalieri \& Agarwal (2014),

$$
\gamma^{2}\left(y_{1}, y_{2}, \omega\right)=\exp \left(-2 \frac{\left(y_{1}-y_{2}\right)^{2}}{L_{c}^{2}(\omega)}\right)
$$

The coherence decay between points $y_{1}$ and $y_{2}$ is now defined by the characteristic coherence length scale $L_{c}$. Introducing this effect by multiplying equations (2.12) and (2.14), we arrive at the CSD of the two-point source model for broadband shockassociated noise

$$
\begin{aligned}
S\left(y_{1}, \omega\right) S^{*}\left(y_{2}, \omega\right)= & A^{2}(\omega) e^{-\left(\frac{y_{1}}{L(\omega)}\right)^{2}} e^{-\left(\frac{y_{2}}{L(\omega)}\right)^{2}}\left\{e^{\mathrm{i} k_{h}(\omega)\left(y_{1}-y_{2}\right)}\right\} e^{\left(-\frac{\left(y_{1}-y_{2}\right)^{2}}{L_{c}(\omega)^{2}}\right)} \times \\
& \sum_{n} c_{s_{n}}\left\{e^{\mathrm{i} k_{s_{n}} y_{1}}+e^{-\mathrm{i} k_{s_{n}} y_{1}}\right\} \sum_{m} c_{s_{m}}\left\{e^{\mathrm{i} k_{s_{m}} y_{2}}+e^{-\mathrm{i} k_{s_{m}} y_{2}}\right\}
\end{aligned}
$$

For a given pair of points $\left(y_{1}, y_{2}\right)$, the source term is described by two wavepacket envelope terms $\exp \left(-y_{1} / L(\omega)\right)^{2}$ and $\exp \left(-y_{2} / L(\omega)\right)^{2}$ at the two points respectively. The term $\exp \left[\mathrm{i} k_{h}(\omega)\left(y_{1}-y_{2}\right)\right]$ describes the phase difference between $y_{1}$ and $y_{2}$ while the coherence decay is modelled by $\exp \left[-\left(y_{1}-y_{2}\right)^{2} / L_{c}^{2}(\omega)\right]$. Finally this is multiplied by the shock-cell modes at points $y_{1}$ and $y_{2}$ by the expression $\sum\left[\exp \left(\mathrm{i} k_{s} y_{1}\right)+\exp \left(-\mathrm{i} k_{s} y_{1}\right)\right] \sum\left[\exp \left(\mathrm{i} k_{s} y_{2}\right)+\right.$ $\left.\exp \left(-\mathrm{i} k_{s} y_{2}\right)\right]$. The frequency dependence notation of $k_{h}, L$ and $L_{c}$, while implied, is now hereafter omitted for convenience.

Similar to Cavalieri \& Agarwal (2014), the model is now governed by two characteristic length scales. The first length scale, $L$, characterises the wavepacket amplitude envelope. The second, $L_{c}$, is the coherence length scale which characterises the decay of coherence between two points along the axial direction. It should be noted that as $L_{c} \rightarrow \infty$, the two-point model (2.15) reduces to the unit-coherence model (2.12). 
The far-field sound pressure for both models can now be found by inserting equations (2.15) and (2.12) into equation (2.10). Using the usual Fraunhofer far-field approximation where $|\boldsymbol{x}-\boldsymbol{y}| \approx|\boldsymbol{x}|-\hat{\mathbf{x}} \cdot \boldsymbol{y}$ (Crighton 1975; Howe 2003), we arrive at the expression

$$
\left\langle p(\boldsymbol{x}, \omega) p^{*}(\boldsymbol{x}, \omega)\right\rangle \approx \frac{A^{2}(\omega)}{4 \pi x^{2}} \iint\left\langle S\left(y_{1}, \omega\right) S^{*}\left(y_{2}, \omega\right)\right\rangle e^{\mathrm{i} k \cos \theta\left(y_{1}-y_{2}\right)} d y_{1} d y_{2},
$$

where $\theta$ is taken as the angle from the downstream jet axis. Due to the line-source approximation for this model, the double volume integral reduces to a double integral in the streamwise direction.

\section{Acoustic efficiency \& directivity}

\subsection{Parameters of the source model}

Our objective here is to study the impact of coherence decay on BBSAN in a model problem. To do so, we must first specify values of the hydrodynamic terms $\left(k_{h}, A(\omega), L\right)$ in equation (2.16). The coherence length term $L_{c}$ must also be specified. The chosen modelling parameters are listed in table 1 and given in Appendix A; the justification of these values is discussed below.

The first parameter, $k_{h}$ is the hydrodynamic wavenumber of the wavepacket defined as $k_{h}=\omega / u_{c}$. We consider the average convection velocity of the wavepackets to be $u_{c} \approx 0.6 U_{j}$, consistent with the literature (Harper-Bourne \& Fisher 1973; Lau 1980; Troutt \& McLaughlin 1982; Kerhervé et al. 2006; Morris \& Zaman 2010).

The second term $A(\omega)$ represents the wavepacket amplitude. While there is no theoretical form for shock-containing flows, it has been previously measured in experimental campaigns (Bridges \& Wernet 2008; Savarese et al. 2013). More recently, Antonialli et al. (2018) were able to determine the frequency dependence of wavepacket amplitudes in a subsonic Mach 0.9 jet by comparing large-eddy simulation data of Brès et al. (2017) to the fluctuation fields predicted from the parabolised stability equations model of Sasaki et al. $(2017 b)$. We therefore model the wavepacket amplitude term using an energy-spectrum function as proposed by Antonialli et al. (2018)

$$
A(\omega)=C_{1} e^{-C_{2} \omega}
$$

where terms $C_{1}$ and $C_{2}$ are fitting parameters with values $3.4 \cdot 10^{-7}$ and 0.58 respectively. The value of $C_{2}$ has been normalised based on the Strouhal number of $S t=f D / U_{j}$. A similar exponential decay spectrum was also used by Suzuki (2016). The numerical value of $A(\omega)$ is obtained by fitting the model (3.1) to the measured velocity spectra (Savarese et al. 2013) obtained along the shear layer at an axial position $y / D \approx 3$ for a jet operating at $N P R=2.5$. The amplitude term is normalised to yield a source strength of unity at the peak frequency.

As we do not have at our disposal the wavepacket length scales $L$ and $L_{c}$ for supersonic jets, we adopt values from previous work on subsonic flows. The objective of this study is not to develop a predictive capability but rather to determine the impact of coherence decay in a model problem. Hence, in the same spirit as Cavalieri \& Agarwal (2014) who used average values independent of frequency, we evaluate the source term in equation (2.16) for $L=1.0 D$ and $k_{h} L=5$. From the two-point measurements of Jaunet et al. (2017) for a $M=0.4$ jet, it is evident that coherence lengths have a frequency and axial position dependence. However, without prior measurements of this dependence in 
(a)

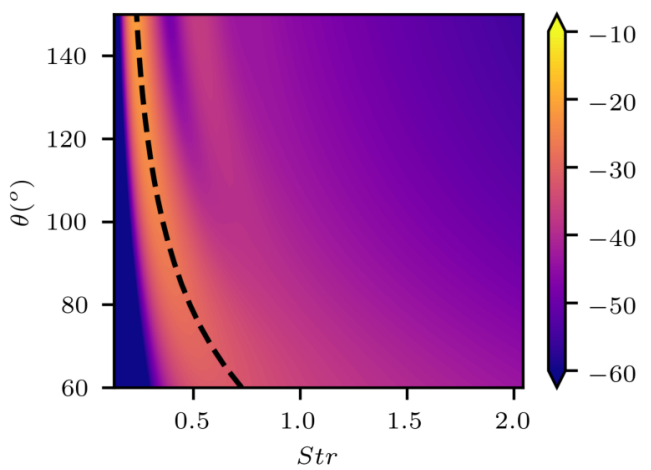

(b)

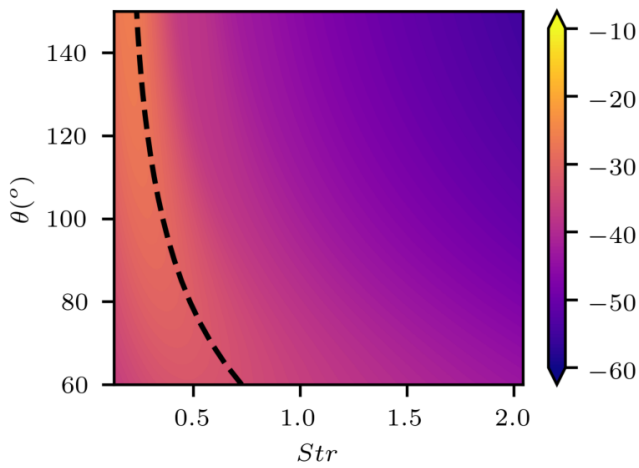

Figure 1. Contours of sound pressure level (arbitrary dB) as a function of frequency $(S t)$ and directivity $(\theta)$ for a) unit coherence and $\mathrm{b}$ ) with coherence decay. The jet issues from a converging nozzle $\left(M_{d}=1.0\right)$ at a nozzle pressure ratio of $N P R=3.4$ which corresponds to a fullyexpanded jet Mach number of $M_{j}=1.45$ and an off-design parameter of $\beta=1.04$. The dashed line indicates the peak frequency as predicted by Harper-Bourne \& Fisher (1973).

shock-containing flows, we adopt for this study an average value of $L_{c}=1.0 \mathrm{D}$ similar to Cavalieri \& Agarwal (2014) and Baqui et al. (2015). It should be noted, however, that Suzuki (2016) did extract the coherence length of the near-field pressure in an underexpanded supersonic jet. An approximately constant coherence length scale was found over a range of frequencies, further suggesting that an average value is suitable for the purposes of this study. The sensitivity of the modelling choices have been investigated with some results presented in Appendix A.

\subsection{Far-field acoustic predictions}

The far-field sound is obtained by numerical evaluation of equation (2.16). Figure 1 shows the variation of far-field SPL as a function of emission angle and frequency for an underexpanded jet operating at $N P R=3.4$. The modelled jet issues from a convergent nozzle, which corresponds to an off-design parameter of $\beta=1.04$. In comparing the case between unit coherence and coherence decay, all tuning parameters as specified in section 3.1 with the exception of $L_{c}$ are kept constant. The far-field sound pressure contours were obtained using the first ten shock-cell modes $(n=10)$. The use of the number of modes is justified in section 5 . It is clear from figure 1 that coherence decay has a significant effect on the BBSAN spectrum. Consistent with experimental observations, both plots comprise a peak frequency that increases with decreasing observation angle; though the effect is more evident in the unit coherence case.

The first stage of the analysis considers cases involving a single shock-cell mode $(n=1)$; the centreline pressure fluctuations in a moderately underexpanded jet are reasonably well represented by a single mode (Tam et al. 1985; Ray \& Lele 2007). Figure 2 shows the directivity for far-field SPL at $S t=0.3$ and $S t=0.6$ for the same conditions as figure 1 but with only the fundamental shock-cell mode $(n=1)$ included. The Strouhal number is defined as $S t=f D / U_{j}$. Models with unit coherence and coherence decay are plotted on the same figure for comparison. At $S t=0.3$, both models predict the highest amplitude of radiation in the direction slightly upstream of perpendicular, consistent with previous findings. At $S t=0.6$, the BBSAN peaks shift downstream but with a smaller sound amplitude. With all other terms equal, the introduction of coherence decay broadens the radiation lobe, increasing the SPL in the downstream direction (low $\theta$ values). Contrary 


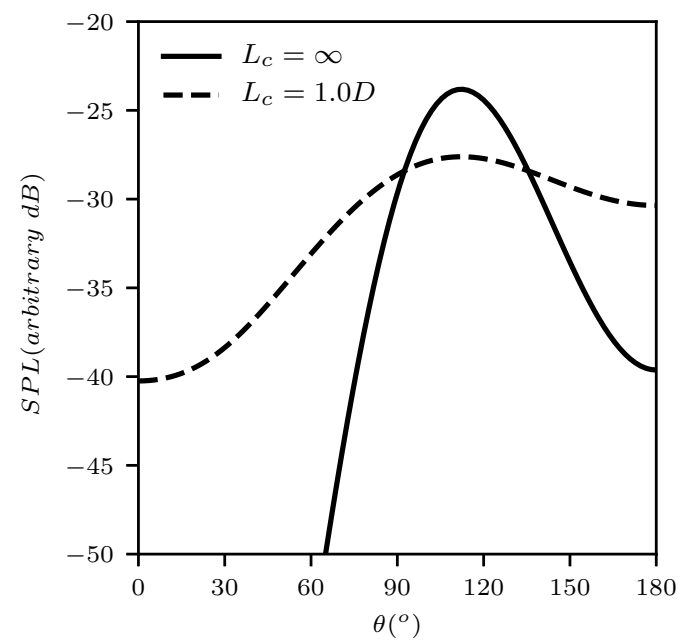

M. H. Wong et al

(b)

Figure 2. Sound pressure level at a distance of $100 D$ for a wavepacket frequency of a) $S t=0.3$ and b) $S t=0.6$ as a function of observation angle $\theta$ for a wavepacket with $k_{h} L=5$.

to the subsonic jet case (Cavalieri et al. 2011; Baqui et al. 2015, amongst others), however, the introduction of coherence decay reduces the peak amplitude by approximately 2-5dB. The reason for this behaviour will be further explored in $\S 4$. It is also evident, from the peak frequency trends in figures 1 and 2 , that the current wavepacket model agrees with the predictions made by localised-phased array models such as Harper-Bourne \& Fisher (1973).

Figure 3 shows a comparison of the noise spectra between the two models and experimental data for an underexpanded jet operating at $N P R=3.4$ (Norum \& Seiner 1982). The modelled peak amplitudes are adjusted to match experimental data in order to facilitate comparison of the spectral shape. As can be seen, while there is reasonable agreement between the two models and the measured data in terms of the peak frequency, the overall agreement between the two models and the data is moderate. Both the unitcoherence and coherence-decay models capture the BBSAN peak frequency dependence and the narrowing of the spectral peak with increasing angle. With the inclusion of coherence decay, however, the BBSAN spectral peak width broadens leading to a more favourable agreement for frequencies greater than the peak. Below $\theta=75^{\circ}$, both models fail to capture the correct peak frequency. A possible explanation for this disagreement in peak frequency at downstream angles could be due to the choice of the $u_{c}$ and $L$ modelling variables as discussed in Appendix A, or the dominance of jet-mixing noise close to the jet axis.

It is evident from figure 3 that the jitter of wavepackets, modelled by coherence decay, broadens the acoustic spectrum. The model suggests, however, that coherence decay does not have a major impact on the sound amplitude. Unlike in subsonic flows, it provides little contribution to the peak SPL. This is also consistent with the results presented by Sinha et al. (2014) for isothermal fully-expanded supersonic jets, where it was found that the far-field noise spectrum at downstream obsevation angles is well captured even without considering the jitter of wavepackets. The reason for this is because in supersonic ideally-expanded flows, wavepackets propagate downstream with supersonic phase velocities. As a result, noise in the form of Mach wave radiation is generated efficiently (Tam 1995) in the downstream direction. On the other hand, in 


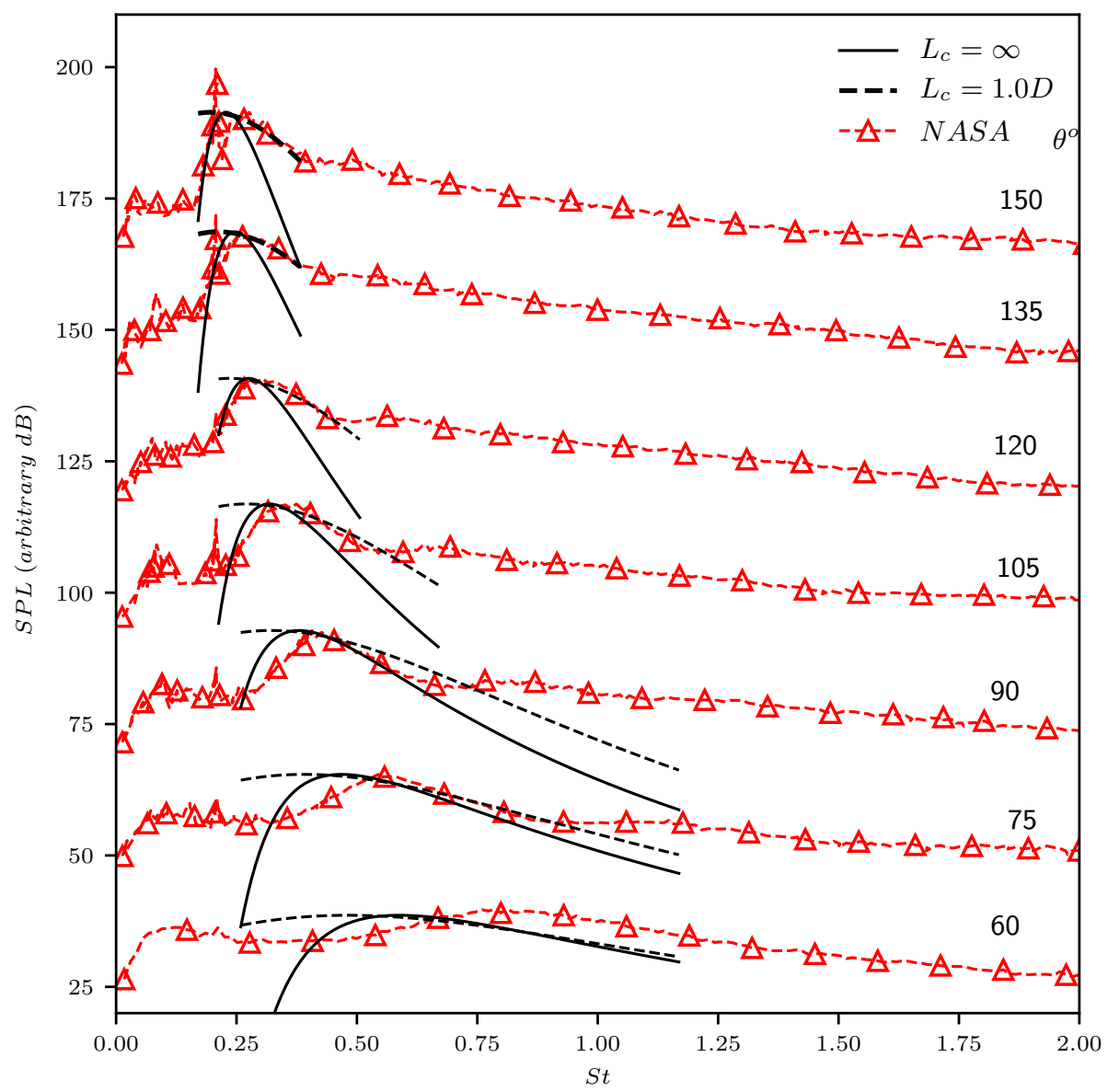

Figure 3. Power spectrum at a distance of $100 D$ through a range of observation angles between $\theta=60^{\circ}$ to $\theta=150^{\circ}$ measured from the downstream jet axis for a wavepacket with $k_{h} L=5$. Each measurement angle is offset by $\Delta d B=25$. NASA experimental data from Norum \& Seiner (1982).

shock-containing flows, the presence of shock-cells generates an additional component which travels upstream. The effect of jittering, on both upstream and downstream travelling components, is discussed in more detail in $\S 4$.

Using a single shock-cell waveguide mode, both wavepacket models show reasonable agreement with the peak of the measured spectrum. However, they suffer from the same issue discussed by Ray \& Lele (2007) where the high frequency sound at upstream angles is 'missing'. In their study, for a $M_{j}=1.22$ isothermal jet, the frequency range of interest was restricted to $S t<1$ due to the assumed breakdown of linear theory at high frequencies. More recently, however, it has been shown by Sasaki et al. $(2017 a)$ that linear theory still yields good agreement for frequencies up to $S t=4$. Therefore, we argue here that the drop-off in high-frequency is not due to the breakdown of linear theory but rather the neglect to include higher-order shock-cell modes. This is further supported by Suzuki's wavepacket model where a similar underestimation of high frequency SPL was observed when using an empirical representation of the shock-cells. The effect of including higher-order modes is discussed in $\S 5$. 


\section{Interpretation of sound radiation characteristics}

\subsection{Fourier transform into wavenumber space}

In order to explore how coherence affects the source structure and sound radiation characteristics, the CSD of the models with and without coherence decay are transformed to wavenumber space. This transformation is achieved with the double Fourier transform,

$$
\mathcal{F}\left(k_{y_{1}}, k_{y_{2}}\right)=\frac{1}{(\sqrt{2 \pi})^{2}} \int_{-\infty}^{\infty} \int_{-\infty}^{\infty} F\left(y_{1}, y_{2}\right) e^{\mathrm{i} k_{y_{1}} y_{1}} e^{\mathrm{i} k_{y_{2}} y_{2}} d y_{1} d y_{2},
$$

where $F\left(y_{1}, y_{2}\right)$ is the two-point expression of the CSD. If we take coherence as unity for the entire domain by inserting equation (2.12) into equation (4.1), the Fourier transform for the perfectly coherent model for a single shock-cell mode is

$$
\begin{aligned}
\mathcal{F}\left(k_{y_{1}}, k_{y_{2}}\right)= & \frac{1}{(\sqrt{2 \pi})^{2}} \int_{-\infty}^{\infty} \int_{-\infty}^{\infty} A_{n=1}^{2}(\omega) e^{-\left(\frac{y_{1}}{L}\right)^{2}} e^{-\left(\frac{y_{2}}{L}\right)^{2}}\left\{e^{\mathrm{i} k_{s} y_{1}}+e^{-\mathrm{i} k_{s} y_{1}}\right\} \times \\
& \left\{e^{\mathrm{i} k_{s} y_{2}}+e^{-\mathrm{i} k_{s} y_{2}}\right\}\left\{e^{\mathrm{i} k_{h}\left(y_{1}-y_{2}\right)}\right\} e^{\mathrm{i} k_{y_{1}} y_{1}} e^{\mathrm{i} k_{y_{2}} y_{2}} d y_{1} d y_{2} .
\end{aligned}
$$

Evaluating the above integral gives

$$
\begin{aligned}
\mathcal{F}\left(k_{y_{1}}, k_{y_{2}}\right)= & \frac{A_{n=1}^{2}(\omega)}{8}\left(e^{-\frac{1}{4}\left(k_{h}-k_{s}+k_{y_{1}}\right)^{2} L^{2}}+e^{-\frac{1}{4}\left(k_{h}+k_{s}+k_{y_{1}}\right)^{2} L^{2}}\right) \times \\
& \left(e^{-\frac{1}{4}\left(k_{h}+k_{s}-k_{y_{2}}\right)^{2} L^{2}}+e^{-\frac{1}{4}\left(-k_{h}+k_{s}+k_{y_{2}}\right)^{2} L^{2}}\right) L^{2}
\end{aligned}
$$

which is the wavenumber spectrum of the perfectly coherent source CSD. Likewise, by inserting equation (2.15) into equation (4.1) and evaluating the resulting integral, the Fourier transformed CSD of the coherence decay model can also be obtained (the solution is presented in Appendix B). Equation (4.3) is obtained from equation (B 1) by taking the limit $L_{c} \rightarrow \infty$.

Both source CSD models in physical space are shown in figure 4a and figure 4b. In this model problem, the jet nozzle is not present and the sources are simply centred at the origin and extended in both positive and negative directions along the jet axis. The peaks in the contour map are similar to the freckled appearance seen in the two-point pressure correlations obtained by Suzuki (2016). As noted by Suzuki, the spacing between the peaks on the diagonal approximately correspond to the shock-cell spacings. The offdiagonal peaks correspond to the wavepacket interacting with adjacent shock-cells. The introduction of coherence decay concentrates the sources in space; along the axis $y_{1}=y_{2}$. This behaviour is also seen in the non-shock containing case, as found by Cavalieri \& Agarwal (2014).

A comparison of the models' Fourier transformed CSDs as given by equation (4.3) and (B 1) is shown in figure 4c and figure 4d respectively. The contour scale is logarithmic and both axes are normalised by the hydrodynamic wavenumber of the wavepacket $k_{h}$. The source term in both cases produces four distinct lobes. The existence and ramifications of these are discussed in detail in $\S 4.2$. The introduction of coherence decay stretches the lobes parallel to the $k_{y 1}=-k_{y 2}$ axis. This is consistent with what has been observed in subsonic jets (Cavalieri \& Agarwal 2014; Jaunet et al. 2017).

As noted by Crighton (1975), only certain spectral components of the source term corresponding to supersonic phase speeds, can contribute to far-field noise. In order to isolate only the radiating wavenumbers of the source term, the following conditions need to be met 
(a)

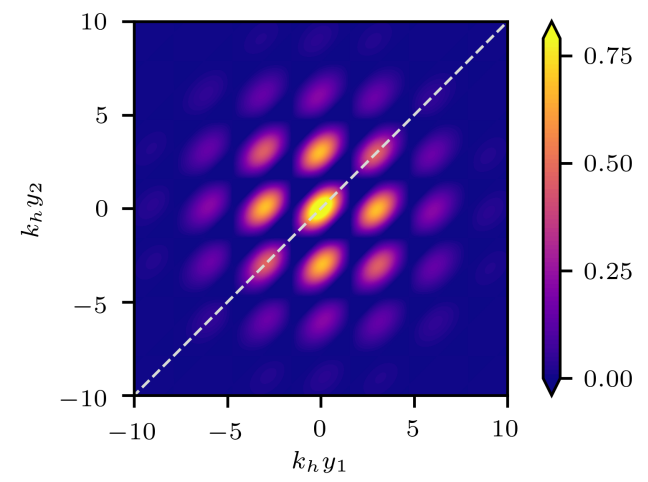

(c)

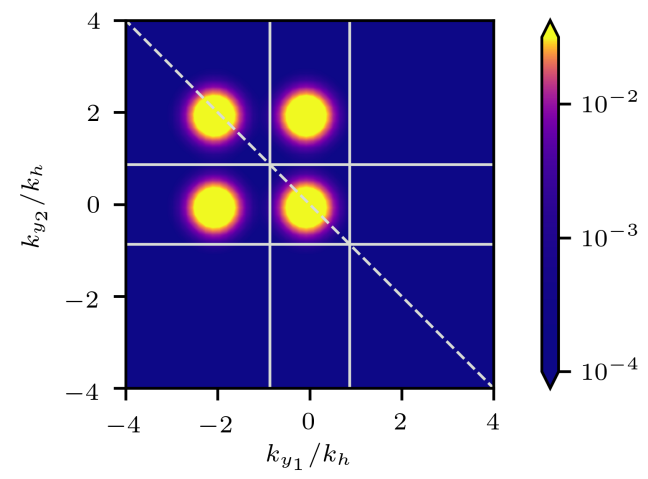

(b)

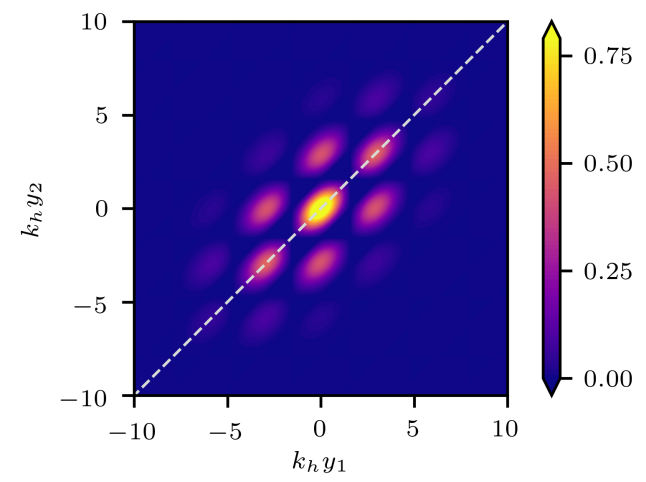

(d)

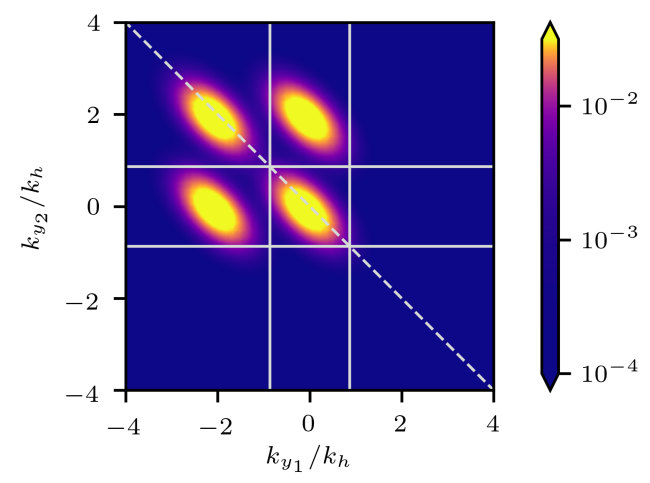

Figure 4. The real part of the CSD of the unit coherence (a) and with statistical decay (b) models for $L_{c}=1.0 D$. The diagonal line represents $y_{1}=y_{2}$. The corresponding Fourier transformed CSD in wavenumber space of the unit coherence (c) and with statistical decay (d) models. Diagonal line corresponds to $k_{y 1}=-k_{y 2}$. The square represents the acoustic matching criterion $|k| / k_{h}=0.6 M_{j}$. The amplitude of both models have been normalised to highlight the effect of coherence decay. The wavepacket frequency is $S t=0.4$ for a jet operating at $\beta=1.04$.

$$
\begin{aligned}
& \frac{\left|k_{y 1}\right|}{k_{h}} \leqslant M_{c} \\
& \frac{\left|k_{y 2}\right|}{k_{h}} \leqslant M_{c},
\end{aligned}
$$

where $M_{c}=\omega /\left(k_{h} c_{0}\right)$ is the convective Mach number. These conditions in wavenumber space are represented by the squares in figure $4 \mathrm{c}$ and $4 \mathrm{~d}$. Source energy that lies outside the square does not contribute to the far-field sound. Unlike the subsonic jet case, where the unit-coherence source lies completely outside the radiation square, the supersonic shock-containing case already has a source lobe satisfying the radiation criterion. This is similar to what is observed in ideally-expanded supersonic jets (Cavalieri \& Agarwal 2014; Sinha et al. 2014). The other three lobes are silent as they lie outside the radiation square.

When coherence decay is introduced we see that the stretching of the radiation lobe 
actually removes a small portion of the source energy from the radiating region. Unlike the subsonic case, where jittering of the wavepacket causes the source energy to be stretched into the radiation square, coherence decay removes energy in the BBSAN case. This explains why the introduction of coherence decay decreases the peak SPL compared to the unit coherence case as seen in figure 2 .

The Fourier transform contour plots can also be used to explain the directivity behaviour observed in figure 2. For a given value of $\theta$, the Fourier-transformed wavenumbers $k_{y_{1}}$ and $k_{y_{2}}$ are given by

$$
\left(\frac{k_{y_{1}}}{k_{h}}, \frac{k_{y_{2}}}{k_{h}}\right)=\left(-M_{c} \cos \theta, M_{c} \cos \theta\right) .
$$

Therefore, for $\theta$ values corresponding to the perpendicular direction, the relevant part of the Fourier transform is close to the origin. Moving away from the origin along this axis will correspond to angles upstream and downstream of the jet axis respectively. Hence, the stretching of the source lobe along the $k_{y_{1}}=-k_{y_{2}}$ axis also broadens the directivity of the jet as depicted in figure 2. This broadening is due to the source energy being stretched in both directions from the origin within the radiation square. To summarise, coherence decay is not a sound amplifier as found in the subsonic case but rather broadens the directivity of BBSAN. This broadening will be seen to be even more important when we consider higher-order shock-cell modes in $\S 5$.

\subsection{Nonlinear interaction terms}

By transforming the CSD from physical space to wavenumber space, it has been demonstrated that not all wavelengths of the line-source model are responsible for sound generation. The source lobes seen in wavenumber space are due to the non-linear interactions present in this BBSAN model. As aforementioned, only source components corresponding to supersonic phase speeds relative to the ambient speed of sound can be effective BBSAN noise generators. In order to test whether the acoustically matched source component is supersonic, we aim to identify the phase speeds of the four source lobes in wavenumber space.

Recall that the kinematic model comprises the multiplicative combination of the wavepacket and the shock-cell structure as described in equation (2.4). Using the unitcoherence case for simplicity, after expanding the shock-cell components, the source term in equation (2.12) can be written as

$$
\begin{aligned}
S\left(y_{1}, \omega\right) S^{*}\left(y_{2}, \omega\right)= & \sum_{n}\left\{e^{\mathrm{i} k_{s}\left(y_{1}+y_{2}\right)}+e^{\mathrm{i} k_{s}\left(y_{1}-y_{2}\right)}+e^{\mathrm{i} k_{s}\left(-y_{1}+y_{2}\right)}+e^{\mathrm{i} k_{s}\left(-y_{1}-y_{2}\right)}\right\} \times \\
& \left\{e^{\mathrm{i} k_{h}\left(y_{1}-y_{2}\right)}\right\},
\end{aligned}
$$

where we have ignored the amplitude and wavepacket envelope terms for this analysis. Expanding again we obtain four terms defined as

$$
S\left(y_{1}, \omega\right) S^{*}\left(y_{2}, \omega\right)=A_{1,2}^{+}+A_{1,2}^{-}+B_{1,2}^{+}+B_{1,2}^{-}
$$

where the terms are shown to be

$$
\begin{aligned}
& A_{1,2}^{+}=e^{i\left(y_{1}-y_{2}\right)\left(k_{h}+k_{s}\right)}, \\
& A_{1,2}^{-}=e^{i\left(y_{1}-y_{2}\right)\left(k_{h}-k_{s}\right)},
\end{aligned}
$$


Impact of coherence decay on wavepacket models for BBSAN in supersonic jets

$$
\begin{aligned}
& B_{1,2}^{+}=e^{i y_{1}\left(k_{s}+k_{h}\right)+i y_{2}\left(k_{s}-k_{h}\right)}, \\
& B_{1,2}^{-}=e^{i y_{1}\left(-k_{s}+k_{h}\right)+i y_{2}\left(-k_{s}-k_{h}\right)} .
\end{aligned}
$$

By grouping the terms in this manner, we see that $A_{1,2}^{+}$and $A_{1,2}^{-}$terms, which are respectively the sum and difference non-linear wave interactions, have phase velocities

$$
\begin{aligned}
& v^{+}=\frac{\omega}{k_{h}+k_{s}}, \\
& v^{-}=\frac{\omega}{k_{h}-k_{s}} .
\end{aligned}
$$

Since $\omega /\left(k_{h}\right)>\omega /\left(k_{h}+k_{s}\right)$, the $A_{1,2}^{+}$component travels slower than the ambient speed of sound and is not acoustically matched. It corresponds to the top left lobe in figure $4 \mathrm{c}$ and $4 \mathrm{~d}$ along the diagonal. Conversely, the term $A_{1,2}^{-}$is capable of either subsonic or supersonic phase speeds and is represented by the bottom right lobe in figure $4 \mathrm{c}$ and $4 \mathrm{~d}$. This explains why only the $A_{1,2}^{-}$component of the total source term is capable of generating far-field noise and is consistent with previous findings (Tam \& Tanna 1982).

The terms $B_{1,2}^{+}$and $B_{1,2}^{-}$, on the other hand, involve combined effects of the sum and difference interaction and are a complex conjugate pair. These terms, however, lie off the $k_{y 1}=-k_{y 2}$ axis and outside the radiation square and therefore do not contribute to the far-field sound. A similar depiction of these off-diagonal lobes are observed in the Fourier transformed CSD maps of Baqui et al. (2015) and non-linear wavenumber interactions in low Reynolds-number subsonic jets as discussed by Sandham et al. (2006).

\section{Coherence decay and higher-order shock-cell modes}

We now focus on the more general model where higher-order shock-cell modes are included. It has been demonstrated that these additional shock-cell modes do make significant contributions to high frequency sound in upstream directions (Tam et al. 1985; Ray \& Lele 2007). We here test the effect of coherence decay when these higher-order shock-cell modes are included.

Tam et al. (1985) have argued that it should only be necessary to include the first four shock-cell modes and any higher modes are unnecessary to describe the shock-cell structure. Ray \& Lele (2007), on the other hand, justified the use of only the fundamental shock-cell mode as source activity associated with higher-order modes lie outside the range of radiating wavenumbers. This is true when coherence decay is not accounted for.

The sensitivity of the acoustic spectrum to the addition of higher-order shock-cell modes was investigated for the current source model. It was found that the far-field spectrum and source structure did not change significantly beyond the first ten modes. Hence only the first ten shock-cell modes, as defined by equation (2.6), were used for this study.

By including higher-order modes, as shown by figure 5, an improvement in spectral shape is observed for all observation angles. Higher waveguide modes are required to describe the acoustic spectra for frequencies greater than the broadband peak, consistent with Tam et al. (1985). While there are still discrepancies for the downstream angle at $\theta=$ $60^{\circ}$, both models predict the peak frequency with reasonable accuracy, though the case with coherence decay slightly under predicts the peak frequency compared to the data. The spectral peak shape predicted when coherence decay is included is more accurate than the case with perfect coherence. For the perfectly coherent wavepacket, oscillations 


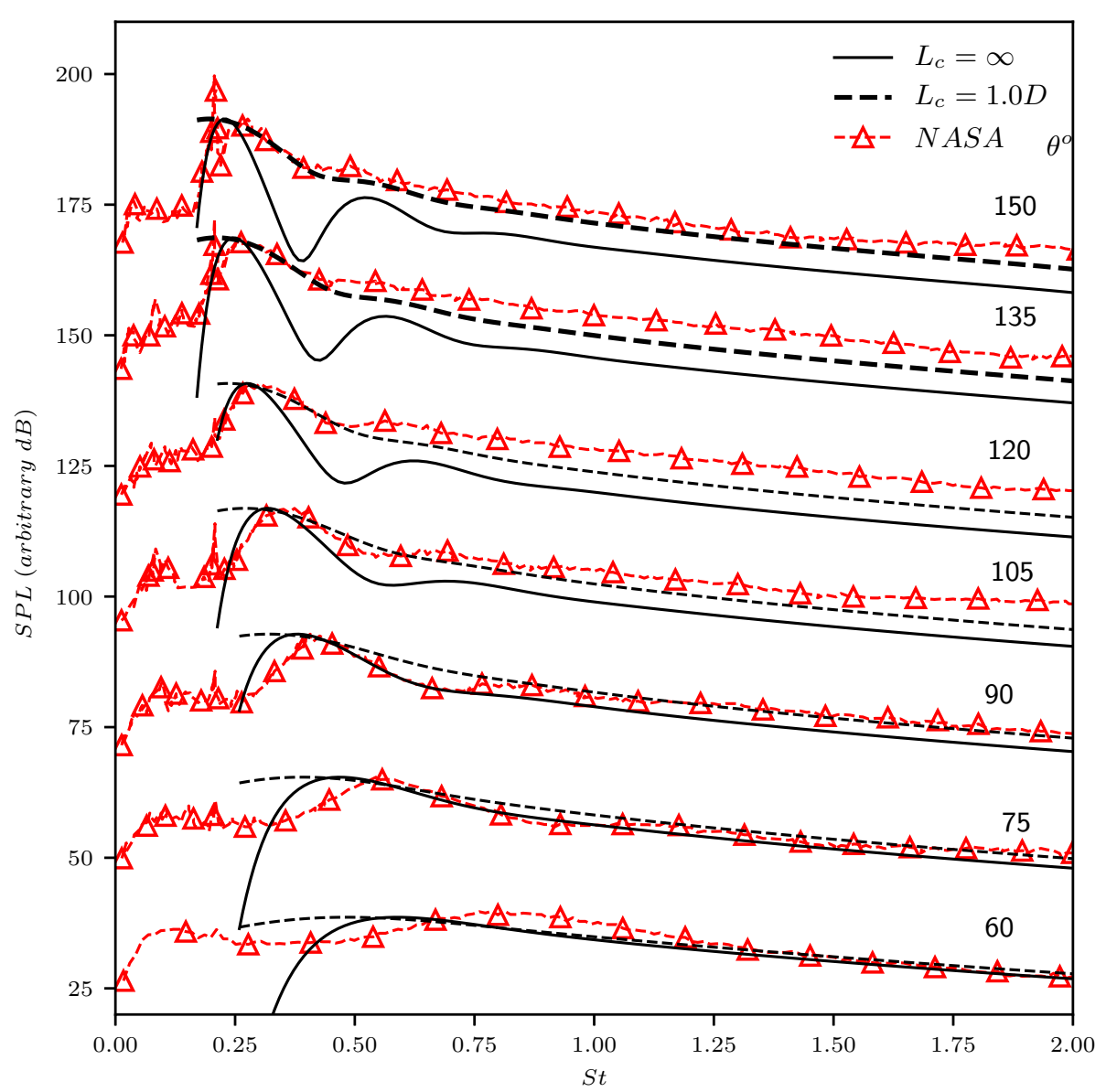

Figure 5. Power spectrum at a distance of $100 D$ through a range of observation angles between $\theta=60^{\circ}$ to $\theta=150^{\circ}$ measured from the downstream jet axis for a wavepacket with $k_{h} L=5$ for multiple shock-cell waveguide modes. Each measurement angle is offset by $25 d B$. NASA experimental data from Norum \& Seiner (1982).

start to occur at higher Strouhal numbers resulting in 'narrow-banded' secondary peaks. This deficiency of the perfectly coherent model at higher frequencies for upstream angles agrees with Ray \& Lele (2007) where it was found that the linear model (unit coherence by construction) becomes 'increasingly suspect at higher frequencies'.

Conversely, the two-point model with coherence decay 'smooths' out these narrowbanded peaks. This observation is consistent with Ray \& Lele's (2007) assertion that non-linear effects can rectify these artificial peaks introduced from the instability wave interacting with the higher-order shock-cell modes. This finding is also consistent with the effect of coherence decay when using localised acoustic sources (Lele 2005). We turn to the Fourier-transformed CSD maps to gain an insight into this behaviour.

The Fourier transformed CSD maps are computed for two frequencies, $S t=0.4$ (figure $6 \mathrm{a}$ and $6 \mathrm{~b}$ ) and $S t=0.6$ (figure $6 \mathrm{c}$ and $6 \mathrm{~d}$ ) where we have now included the first ten shock-cell waveguide modes. Compared to the previous single-mode $(n=1)$ case, additional lobes are now visible throughout the wavenumber domain. The additional source-energy lobes are due to the wavepackets interacting with the higher-order Fourier shock-cell modes. 
(a)

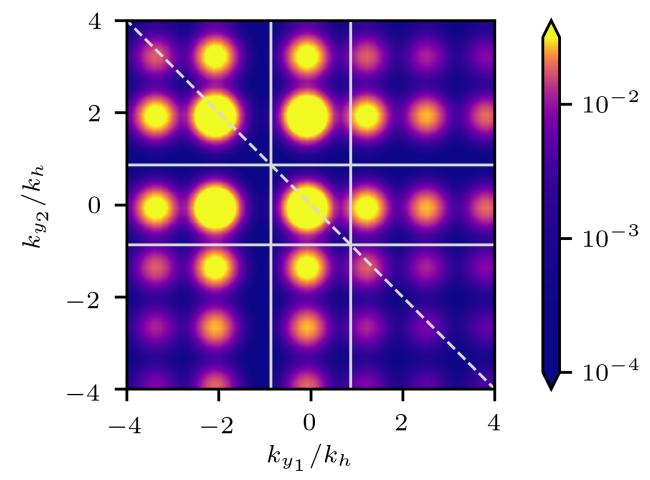

(c)

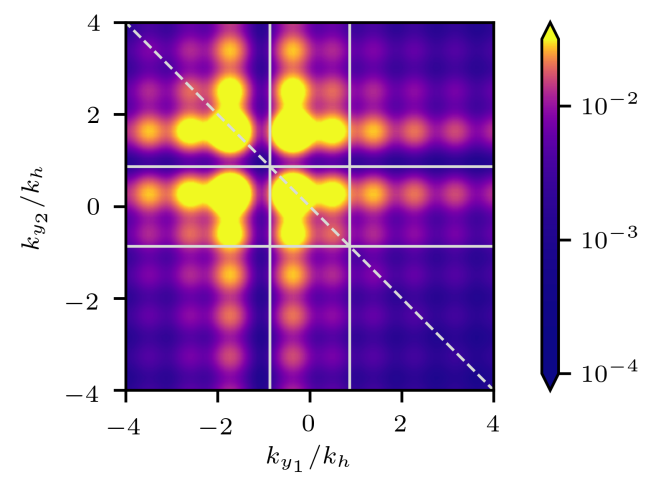

(b)

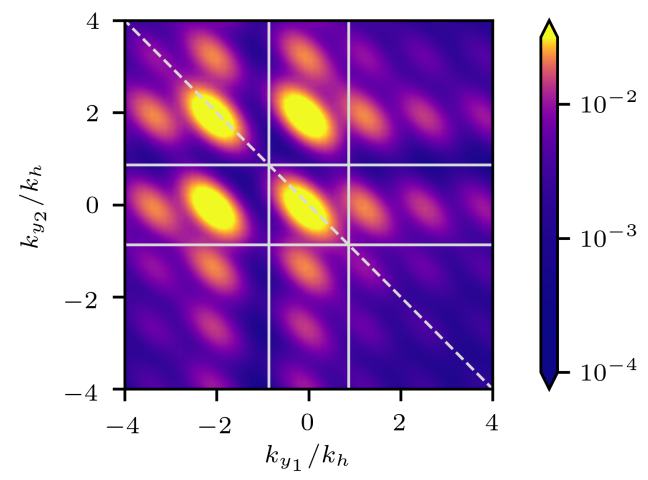

(d)

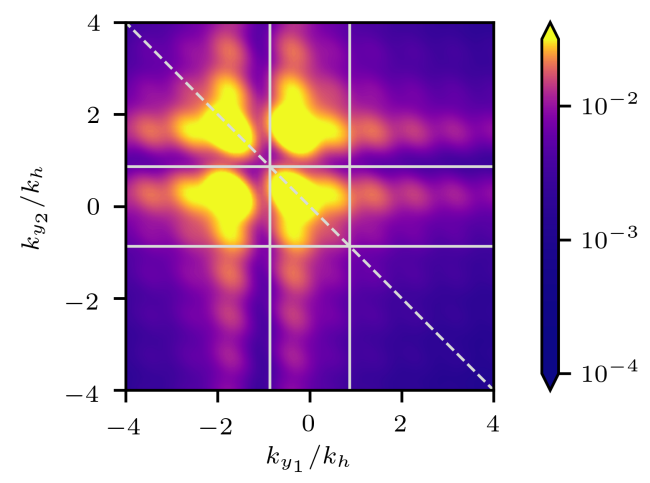

Figure 6. The corresponding Fourier transformed CSD in wavenumber space for $S t=0.4$ and $S t=0.6$. Left-hand side frames ( (a) and (c)) correspond to the unit coherence model while the right-hand side ((b) and (d)) are for the two-point model with a coherence decay length $L_{c}=$ 1.0D. Diagonal line corresponds to $k_{y_{1}}=-k_{y_{2}}$. The square represents the acoustic matching criterion $|k| / k_{h}=0.6 M_{j}$. The amplitude of both models have been normalised to highlight the effect of coherence decay. The jet is operating at $\beta=1.04$.

For the unit-coherence case (figure $6 \mathrm{a}$ and $6 \mathrm{c}$ ), the far-field sound radiation is still dominated by the wavepacket interaction with the $n=1$ waveguide mode. For $S t=0.4$, interaction with the second mode does not contribute to the far-field radiation as the source energy from this interaction lies outside the radiation square. This is what was observed by Ray \& Lele (2007) and it was the reason modes higher than the fundamental were not considered in their study. At a higher frequency $(S t=0.6)$, however, the Fourier lobes become more compact and the $n=2$ modes migrate into the radiation square as seen in figure $6 \mathrm{c}$. This is consistent with the behaviour seen in the far-field acoustic spectrum of figures 3 and 5 . For frequencies below the broadband peak, we do not see an increase in SPL when higher-order shock modes are added.

For the model where coherence decay is taken into account (figure $6 \mathrm{~b}$ and $6 \mathrm{~d}$ ), we observe once again a stretching of the source lobes parallel to the $k_{y_{1}}=-k_{y_{2}}$ line. The stretching causes the fundamental $(n=1)$ mode to merge with the second mode and spreads the source energy within the radiating square. In contrast to the unit-coherence case, the $n=2$ shock-cell mode is now also responsible for far-field sound production. 
(a)

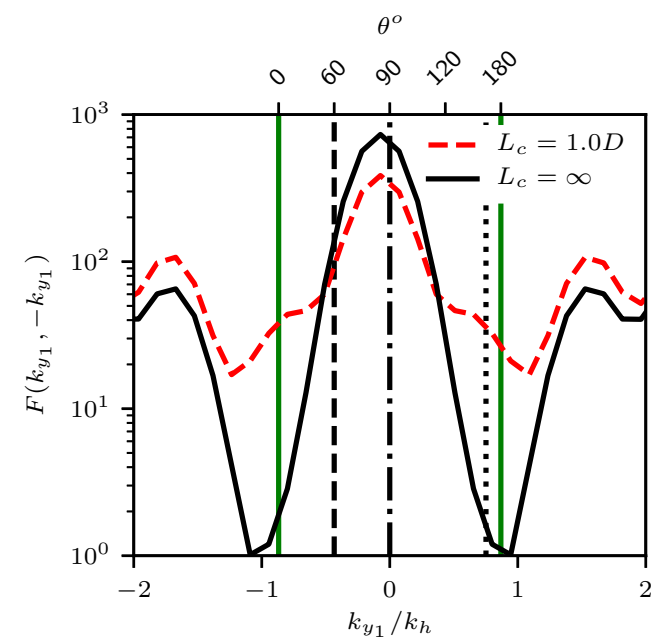

M. H. Wong et al

(b)

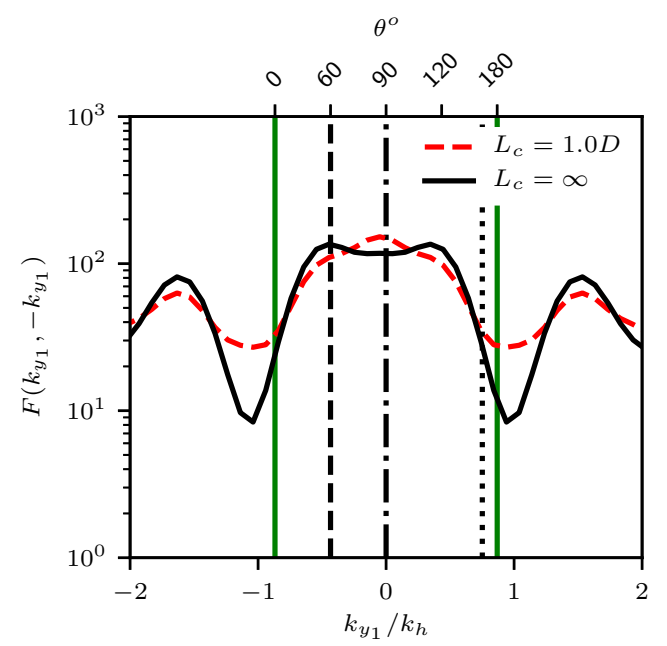

Figure 7. Fourier transform of CSD for a) $S t=0.4$ and b) $S t=0.6$ extracted along the line $k_{y_{1}}=-k_{y_{2}}$. The vertical lines represent different observation angles; dashed line $=60^{\circ}$, dashdotted line $=90^{\circ}$ and dotted line $=150^{\circ}$. Solid (green) vertical lines represent the acoustic matching criterion.

This means that coherence decay, which accounts for stochastic effects, can rectify the 'missing sound' at higher frequencies in the upstream direction seen in figure 3 . It is clear, when compared to the perfectly coherent wavepacket, coherence decay 'spreads and smooths' the source energy in wavenumber space. This leads to a more uniform farfield spectrum for all observation angles and indeed, a smoother directivity also results. This levelling effect in both directivity and frequency is evident when comparing the SPL contour plots in figure $1 \mathrm{a}$ and figure 1b. Nevertheless, the overprediction in SPL for frequencies below the spectral peak is still present for all observation angles. The overprediction at low frequencies is most likely a result of the ad-hoc constant coherence length assumption.

Suzuki (2016) has also incorporated the effect of coherence decay of wavepackets in his model. However, the empirical treatment of the shock-cells as Gaussian humps does not allow the afore-discussed effects of coherence decay to be observed. Similar to Ray \& Lele (2007), the loss of high frequency sound can be attributed to the lack of representation of the higher order shock-cell modes. The predictions from both Suzuki's and Ray \& Lele's models are closer to those of figure 3 and hence lack the same agreement to experimental data as other phased-array models such as Harper-Bourne \& Fisher (1973) and Morris \& Miller (2010).

To show the impact of coherence decay on noise directivity more clearly, the Fourier transformed points along the $k_{y_{1}}=-k_{y_{2}}$ line are extracted and shown as dashed lines in figure $7 \mathrm{a}$ and $7 \mathrm{~b}$. Vertical lines corresponding to different radiation directions as specified by equation (4.5) are also shown for reference. For frequencies slightly greater than the BBSAN peak $(S t=0.4)$, coherence decay amplifies the radiated sound in the upstream direction and smooths out the artificial peaks seen in the perfectly coherent case. On the other hand, for higher frequencies $(S t=0.6)$, the second shock-cell mode is now also contributing to the far-field sound and the effect of coherence decay is minimal. Comparing this to linear instability model results from Ray \& Lele (2007), the impact of coherence decay on higher frequencies is now apparent. 
Due to the ad-hoc nature of fixing the modelling parameters to constant values, it is important to establish that changing the combinations and values does not lead to different conclusions. This is done in Appendix A. We find, amongst other effects, that the suppression of the narrow-banded peaks cannot be accounted for by the tuning of $u_{c}, A(\omega)$ and $L$ within values realistic for supersonic underexpanded jets. We conclude that the inclusion of coherence decay, in the wavepacket framework, is thus imperative. In order to improve predictions at higher frequencies for BBSAN, not only do we need high-order shock modes but also the stochastic forcing term, which in the kinematic model is represented by coherence decay.

The importance of coherence in BBSSAN source modelling has been well-recognised since Harper-Bourne \& Fisher's original model. Using the present BBSAN wavepacket model, however, we suggest that the overall two-point coherence contains much nonacoustically efficient information and that only the coherence decay of the low-order azimuthal modes (wavepackets) is critical in predicting far-field sound. This further suggests a mechanistic explanation for coherence decay in these systems: the coherence decay across sound sources of existing BBSAN models results from the jittering of wavepackets (Williams \& Kempton 1978; Cavalieri et al. 2011).

\section{Conclusions and perspectives}

Motivated by the pioneering work of Tam (1987), the BBSAN models proposed by Ray \& Lele (2007) and Suzuki (2016) indicate the suitability of using wavepackets for this component of jet noise. While successful in predicting many of the known features, the simplified models could not accurately capture the high-frequency sound produced at upstream angles. This is in contrast to the largely accurate predictions made by wavepacket models for supersonic ideally-expanded flows (Sinha et al. 2014). Wavepacket modelling of subsonic jets suggest that coherence decay of the large-scale structures is critical in those flows. Previous BBSAN models (Harper-Bourne \& Fisher 1973; Morris \& Miller 2010) have demonstrated the importance of two-point coherence (or correlation), but only considered it in the context of bulk-turbulent statistics. By constructing a twopoint wavepacket model, using the same methodology as Cavalieri \& Agarwal (2014), we test the impact of wavepacket coherence decay in shock-containing flows.

The semi-empirical kinematic model presented here provides physical insights into the underlying flow physics: the demonstration that wavepacket jitter is central to the sound generation process in shock-containing flows. The model allows us to test the hypothesis of the suitability in using low-order azimuthal structures with coherence decay to predict BBSAN.

By transforming the single-point model of Lele (2005) into a two-point framework, we show that coherence decay may be crucial in predicting higher frequency noise in the upstream direction. Unlike in subsonic jets, the inclusion of coherence decay for BBSAN decreases the acoustic efficiency of wavepackets at peak frequency, but spreads source energy over a greater directivity range.

More significantly, however, is the finding that wavepacket jitter is vital for predicting frequencies above the peak. By capturing this jitter as coherence decay, clear improvements in prediction accuracy are made, especially in the upstream and sideline directions where BBSAN dominates. As exemplified in the results of $\S 5$, coherence decay enables sound generation from higher-order shock-cell modes by stretching the source energy into the radiating square.

In addition to offering insight into the physical mechanisms of shock noise, these results also suggest directions for future modelling efforts. The combined effect of co- 
herence decay and higher-order shock-cell modes need to be incorporated into a dynamic modelling approach that obtains wavepackets and shock-cell modes from linear stability calculations. We show that such calculations would need to be forced stochastically, to produce jittering solutions with coherence decay, and they would be required to include multiple shock-cell modes. The findings from this study are expected to help guide both future kinematic and dynamic wavepacket models of BBSAN.

\section{Acknowledgements}

This work was supported by the Australian Government via a Research Training Program (RTP) Scholarship and the Australian Research Council through the Discovery Projects scheme. The authors would also like to thank Ms. Rhiannon Kirby for her diligent checking of the mathematical formulation.

\section{Appendix A. Parametric Study of Model Parameters}

In this appendix we present results from a short parametric study on the impact of the different model parameters as listed in $\S 3.1$. In order to evaluate each of its effects on the far-field acoustic spectrum, we adjust each parameter individually. When not varied, each parameter is kept constant with the values used equivalent to those stated in $\S 3.1$ and summarised in table 1 . The value of $C_{1}$ from equation 3.1 was kept constant as this would only affect the overall amplitude of the noise generated. We show in figure 8 the results for an observation angle $\theta=150^{\circ}$.

As expected, we notice that varying the values of each modelling parameter changes the shape of the acoustic spectrum produced. Increasing the convection velocity $u_{c}$ shifts the peak frequency to higher Strouhal values as shown in figure 8a. The rapid dropoff at high Strouhal values from increasing $C_{2}$ in figure $8 \mathrm{~b}$ corresponds to the growing exponential decay as $C_{2}$ increases. While it can be seen that $u_{c}$ and $C_{2}$ does alter the far-field sound spectrum, their effects on the harmonic peaks remain minimal.

We note, however, the two length scales characterising the wavepacket do have a more significant effect on the higher-order peaks. From figures 8c and 8d, neither $L$ nor $L_{c}$ alters the peak frequency predicted. We note that for a large spatial wavepacket envelope $(L)$ the peak sound amplitude increases but the artificial peaks become more apparent. As we decrease the value of $L$, the peaks are suppressed; similar to the impact we see with using a finite value of $L_{c}$ in figure 8d.

To further investigate the relationship between $L$ and $L_{c}$ and their effect with respect to directivity, a set of sound pressure contours is presented in figure 9 . The dependency of peak frequency on directivity is lost for small values of $L$ and $L_{c}$. Increasing $L$ seems to make the BBSAN peak more prominent for all directivity angles and is consistent with previous findings on the effect of spatial modulation of wavepackets (Cavalieri et al. 2011, 2012). The non-compact nature of wavepackets explains, for example, the axisymmetric superdirective radiation of low Mach number turbulent jets (Laufer 1983). In supersonic flows, this can be seen in the directivity dependence of the peak sound-emission frequency.

As found by Cavalieri et al. (2012), for superdirectivity to occur, the wavepacket source must not be compact; the condition of $k_{h} L>>1$ must be met. For BBSAN, the peak frequency also exhibits a directivity dependence (Harper-Bourne \& Fisher 1973). Hence, even though by decreasing the value of $L$ we get the same effect as including coherence decay, we find that the values which would be found in supersonic shock-containing jets will not be small. Using two-point correlations of near-field pressure from LES data, 
Suzuki (2016) also found that the axial extent of wavepackets extended over several jet diameters.

While narrow wavepacket spatial envelopes have been shown in figure $8 \mathrm{c}$ to suppress the higher-order peaks, the loss of peak frequency directivity (figure 9) and results from previous studies demonstrate that these small-narrow wavepacket envelopes are not plausible. In shock-containing flows, wavepackets are non-compact. Instead, as discussed in section 5, we propose that coherence decay is responsible for smoothing out these higher-order peaks.

$\begin{array}{lc}\text { Parameter } & \text { Value } \\ N P R & 3.4 \\ u_{c} & 0.6 U_{j} \\ C_{2} & 0.58 \\ k_{h} L & 5.0 \\ L_{c} & 1.0 \mathrm{D}\end{array}$

Table 1. Constant values for each parameter used in proposed model.

(a)

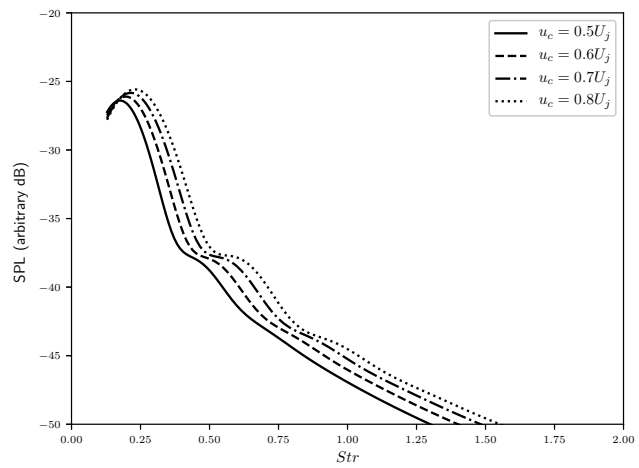

(c)

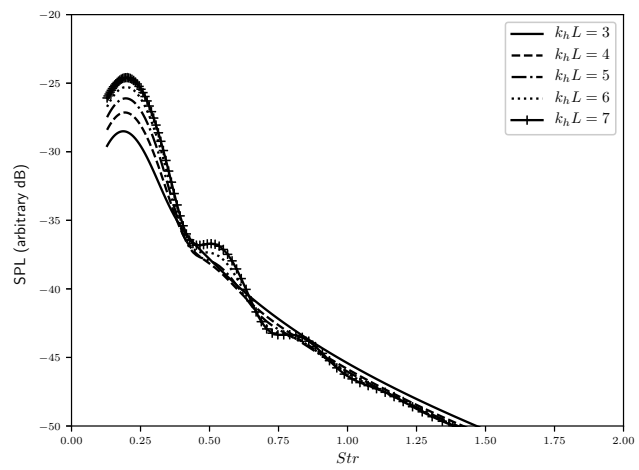

(b)

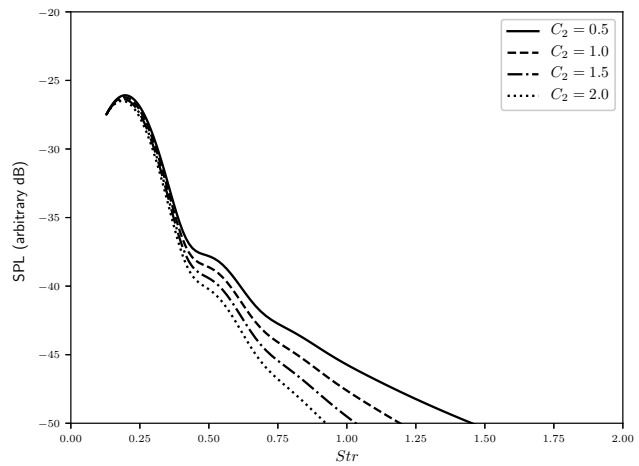

(d)

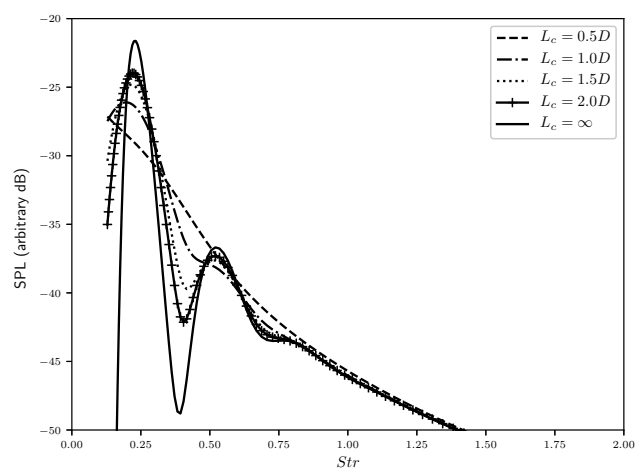

Figure 8. Effect of modelling variables on the acoustic power spectrum when (a) changing convection velocity $u_{c}$, (b) changing amplitude width $C_{2}$, (c) changing wavepacket envelope length scale $L,(\mathrm{~d})$ changing coherence decay length scale $L_{c}$ for an observation angle of $\theta=150^{\circ}$ at a distance of $100 D$. The modelled jet is operating at a nozzle pressure ratio of $N P R=3.4$. 
(a)

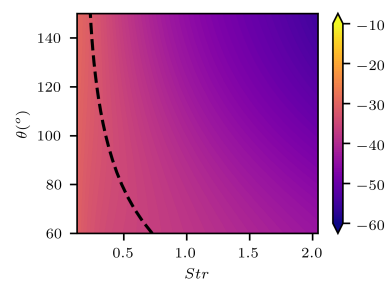

(d)

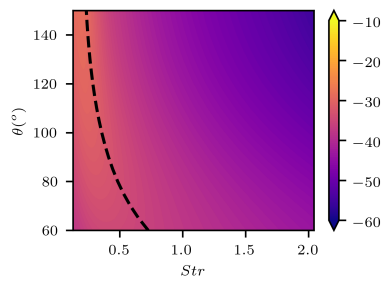

(g)

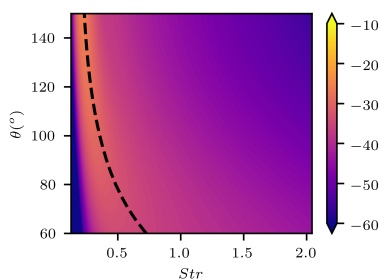

(b)

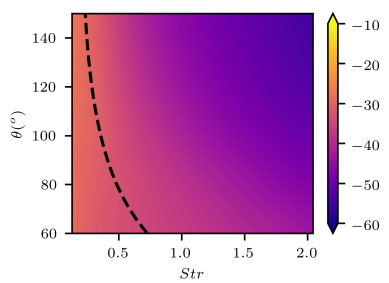

(e)

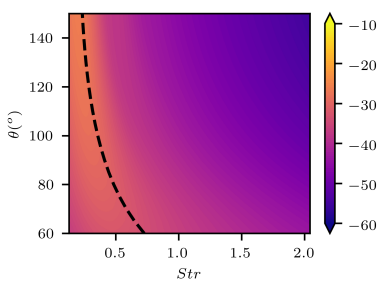

(h)

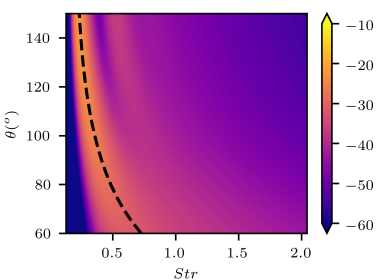

(c)

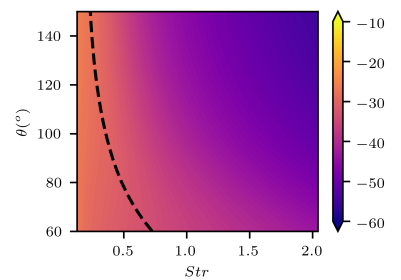

(f)

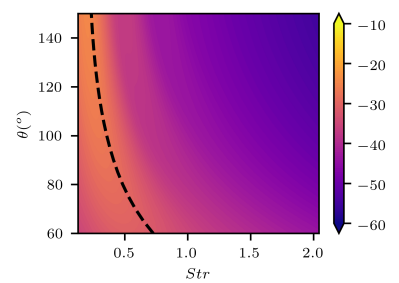

(i)

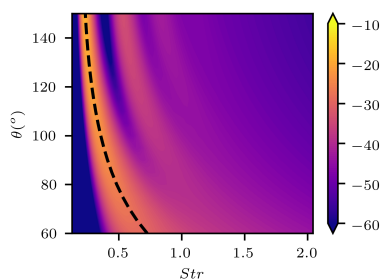

Figure 9. Contours of sound pressure level (arbitrary $\mathrm{dB}$ ) as a function of frequency $(S t)$ and directivity $(\theta)$ for a range of $L_{c}$ and $L$. Each row corresponds to the two-point model with constant value of $L_{c}$; a)-c) $L_{c}=0.5 D$, d)-f) $L_{c}=1.0 D$, g)-i) $L_{c}=\infty$. Each column corresponds to the two-point model with constant value of $\left.k_{h} L ; k_{h} L=3.0(\mathrm{a}), \mathrm{d}\right)$ and $\left.\mathrm{g}\right)$ ), $\left.k_{h} L=5.0(\mathrm{~b}), \mathrm{e}\right)$ and $\mathrm{h})$ ) and $\left.k_{h} L=7.0(\mathrm{c}), \mathrm{f}\right)$ and i)). The modelled jet is operating at a nozzle pressure ratio of $N P R=3.4$.

\section{Appendix B. Fourier transform of two-point CSD}

We present below the result from carrying out the double Fourier transform of equation (2.15).

$$
\begin{aligned}
& \mathcal{F}\left(k_{y_{1}}, k_{y_{2}}\right)=\frac{1}{8 \sqrt{\frac{1}{L^{2}}+\frac{1}{L_{c}^{2}}} \sqrt{\frac{2 L^{2}+L_{c}^{2}}{L^{4}+L^{2} L_{c}^{2}}}}( \\
& e^{-\frac{L^{2}\left(k_{z}^{2} L^{2}+2 k_{h}^{2} L_{c}^{2}-2 k_{h} k_{z} L_{c}^{2}+k_{z}^{2} L_{c}^{2}+k_{y}^{2}\left(L^{2}+L_{c}^{2}\right)+2 k_{s}^{2}\left(2 L^{2}+L_{c}^{2}\right)-2 k_{s}\left(k_{y}+k_{z}\right)\left(2 L^{2}+L_{c}^{2}\right)+2 k_{y}\left(k_{z} L^{2}+k_{h} L_{c}^{2}\right)\right)}{4\left(2 L^{2}+L_{c}^{2}\right)}+}+ \\
& e^{-\frac{L^{2}\left(k_{z}^{2} L^{2}+2 k_{h}^{2} L_{c}^{2}-2 k_{h} k_{z} L_{c}^{2}+k_{z}^{2} L_{c}^{2}+k_{y}^{2}\left(L^{2}+L_{c}^{2}\right)+2 k_{s}^{2}\left(2 L^{2}+L_{c}^{2}\right)+2 k_{s}\left(k_{y}+k_{z}\right)\left(2 L^{2}+L_{c}^{2}\right)+2 k_{y}\left(k_{z} L^{2}+k_{h} L_{c}^{2}\right)\right)}{4\left(2 L^{2}+L_{c}^{2}\right)}}+ \\
& e^{-\frac{L^{2}\left(2\left(k_{h}-k_{s}\right)^{2} L_{c}^{2}+2\left(-k_{h}+k_{s}\right) k_{z} L_{c}^{2}+k_{y}^{2}\left(L^{2}+L_{c}^{2}\right)+k_{z}^{2}\left(L^{2}+L_{c}^{2}\right)+2 k_{y}\left(k_{z} L^{2}+\left(k_{h}-k_{s}\right) L_{c}^{2}\right)\right)}{4\left(2 L^{2}+L_{c}^{2}\right)}}+
\end{aligned}
$$




\section{REFERENCES}

Antonialli, la, Cavalieri, AV, Schmidt, OT, Colonius, T, Jordan, P, Towne, A, BrÉs, G, \& Agarwal, A 2018 Amplitude scaling of turbulent-jet wavepackets. In 23rd AIAA/CEAS Aeroacoustics Conference, p. under review.

Baqui, YB, Agarwal, A, Cavalieri, AVG \& Sinayoko, S 2013 Nonlinear and linear noise source mechanisms in subsonic jets. In 19th AIAA/CEAS Aeroacoustics Conference, AIAA Paper, , vol. 2087.

Baqui, YB, Agarwal, A, Cavalieri, AVG \& Sinayoko, S 2015 A coherence-matched linear source mechanism for subsonic jet noise. Journal of Fluid Mechanics 776, 235-267.

Breakey, DE, Jordan, P, CAvalieri, AVG \& LÉon, O 2013 Near-field wavepackets and the far-field sound of a subsonic jet. In 19th AIAA/CEAS aeroacoustics conference, p. 2083.

Brès, GA, HAm, FE, Nichols, JW \& Lele, SK 2017 Unstructured large-eddy simulations of supersonic jets. AIAA Journal pp. 1164-1184.

BRIDGES, J \& WERNET, MP 2008 Turbulence associated with broadband shock noise in hot jets. In 14th AIAA/CEAS Aeroacoustics Conference (29th AIAA Aeroacoustics Conference), p. 2834.

CAvalieri, AVG \& Agarwal, A 2014 Coherence decay and its impact on sound radiation by wavepackets. Journal of Fluid Mechanics 748, 399-415.

Cavalieri, AVG, Jordan, P, Agarwal, A \& Gervais, Y 2011 Jittering wave-packet models for subsonic jet noise. Journal of Sound and Vibration 330 (18), 4474-4492.

Cavalieri, AVG, Jordan, P, Colonius, T \& Gervais, Y 2012 Axisymmetric superdirectivity in subsonic jets. Journal of fluid Mechanics 704, 388-420.

Cavalieri, AVG, Jordan, P, Wolf, WR \& Gervais, Y 2014 Scattering of wavepackets by a flat plate in the vicinity of a turbulent jet. Journal of sound and Vibration 333 (24), 6516-6531.

Cavalieri, AVG, Rodríguez, D, Jordan, P, Colonius, T \& Gervais, Y 2013 Wavepackets in the velocity field of turbulent jets. Journal of fluid mechanics $\mathbf{7 3 0}, 559-592$.

Cheung, LC \& LeLe, SK 2009 Linear and nonlinear processes in two-dimensional mixing layer dynamics and sound radiation. Journal of Fluid Mechanics 625, 321-351.

Crighton, DG 1975 Basic principles of aerodynamic noise generation. Progress in Aerospace Sciences 16 (1), 31-96.

Crow, S Cu \& Champagne, FH 1971 Orderly structure in jet turbulence. Journal of Fluid Mechanics 48 (3), 547-591.

Edgington-Mitchell, D, Oberleithner, K, Honnery, DR \& Soria, J 2014 Coherent structure and sound production in the helical mode of a screeching axisymmetric jet. Journal of Fluid Mechanics 748, 822-847.

Freund, JB 2003 Noise-source turbulence statistics and the noise from a mach 0.9 jet. Physics of Fluids 15 (6), 1788-1799.

Gudmundsson, K \& Colonius, T 2011 Instability wave models for the near-field fluctuations of turbulent jets. Journal of Fluid Mechanics 689, 97-128.

HARPER-Bourne, MARCus 2002 On modelling the near-field noise of the high-speed jet exhausts of combat aircraft. In 8th AIAA/CEAS Aeroacoustics Conference ES Exhibit, p. 2424.

HARPER-Bourne, M \& Fisher, MJ 1973 The noise from shock waves in supersonic jets. AGARD-CP-131 11, 1-13.

Howe, MS 2003 Theory of vortex sound, , vol. 33. Cambridge University Press.

Jaunet, V, Jordan, P \& CAVAlieri, AVG 2017 Two-point coherence of wave packets in turbulent jets. Physical Review Fluids 2 (2), 024604.

Jordan, P \& Colonius, T 2013 Wave packets and turbulent jet noise. Annual Review of Fluid Mechanics 45, 173-195.

Jordan, P, Colonius, T, Bres, GA, Zhang, M, Towne, A \& Lele, SK 2014 Modeling intermittent wavepackets and their radiated sound in a turbulent jet. In Proceedings of the Summer Program, p. 241. Center for Turbulence Research, Stanford University.

Jordan, P \& GervaIs, Y 2005 Modelling self-and shear-noise mechanisms in inhomogeneous, anisotropic turbulence. Journal of sound and vibration 279 (3-5), 529-555.

Kalyan, A \& Karabasov, SA 2017 Broad band shock associated noise predictions in axisymmetric and asymmetric jets using an improved turbulence scale model. Journal of Sound and Vibration 394, 392-417. 
Kerhervé, F, Fitzpatrick, J \& Jordan, P 2006 The frequency dependence of jet turbulence for noise source modelling. Journal of sound and vibration 296 (1-2), 209-225.

Kerhervé, F, Jordan, P, Gervais, Y, Valiere, JC \& Braud, P 2004 Two-point laser doppler velocimetry measurements in a mach 1.2 cold supersonic jet for statistical aeroacoustic source model. Experiments in fluids 37 (3), 419-437.

Landahl, Mårten T \& Mollo-Christensen, E 1992 Turbulence and random processes in fluid mechanics. Cambridge University Press.

LAU, JC 1980 Laser velocimeter correlation measurements in subsonic and supersonic jets. Journal of sound and vibration 70 (1), 85-101.

LAUfer, JAND Yen, TC 1983 Noise generation by a low-mach-number jet. Journal of Fluid Mechanics 134, 1-31.

LELE, SK 2005 Phased array models of shock-cell noise sources. AIAA Paper 2841, 2005.

LÉON, O \& BRAZIER, JP 2013 Investigation of the near and far pressure fields of dual-stream jets using an euler-based pse model. In 19th AIAA/CEAS Aeroacoustics Conference, p. 2280.

Lighthill, MJ 1952 On sound generated aerodynamically. i. general theory. In Proceedings of the Royal Society of London A: Mathematical, Physical and Engineering Sciences, , vol. 211, pp. 564-587. The Royal Society.

Maia, IA, Jordan, P, Jaunet, V \& Cavalieri, AVG 2017 Two-point wavepacket modelling of jet noise. In 23rd AIAA/CEAS Aeroacoustics Conference, p. 3380.

MichalKe, A 1970 A wave model for sound generation in circular jets .

Miller, SAE \& MORRIS, PJ 2010 The prediction of broadband shock-associated noise from dualstream and rectangular jets using rans cfd. In 16th AIAA/CEAS Aeroacoustics Conference, , vol. 31, pp. 49-56.

Mollo-Christensen, E 1967 Jet noise and shear flow instability seen from an experimenter's viewpoint. Journal of Applied Mechanics 34 (1), 1-7.

Morris, PJ \& Miller, SAE 2010 Prediction of broadband shock-associated noise using reynolds-averaged navier-stokes computational fluid dynamics. AIAA journal 48 (12), 2931.

Morris, PJ \& ZAMAN, KBMQ 2010 Velocity measurements in jets with application to noise source modeling. Journal of sound and vibration 329 (4), 394-414.

Norum, TD \& SEINER, JM 1982 Measurements of mean static pressure and far field acoustics of shock containing supersonic jets .

PACK, DC 1950 A note on prandtl's formula for the wave-length of a supersonic gas jet. The Quarterly Journal of Mechanics and Applied Mathematics 3 (2), 173-181.

PokorA, CD \& MCGuirk, JJ 2015 Stereo-piv measurements of spatio-temporal turbulence correlations in an axisymmetric jet. Journal of Fluid Mechanics 778, 216-252.

Powell, A 1953 On the mechanism of choked jet noise. Proceedings of the Physical Society. Section B 66 (12), 1039.

PrandtL, L 1904 Über die stationären Wellen in einem Gasstrahl. Hirzel.

RAY, PK \& LELE, SK 2007 Sound generated by instability wave/shock-cell interaction in supersonic jets. Journal of fluid mechanics 587, 173-215.

Sandham, ND, Morfey, CL \& Hu, ZW 2006 Nonlinear mechanisms of sound generation in a perturbed parallel jet flow. Journal of Fluid Mechanics 565, 1-23.

Sasaki, K, Cavalieri, AVG, Jordan, P, Schmidt, OT, Colonius, T \& Brès, GA $2017 a$ High-frequency wavepackets in turbulent jets. Journal of Fluid Mechanics $\mathbf{8 3 0}$.

Sasaki, K, Piantanida, S, Cavalieri, AVG \& Jordan, P $2017 b$ Real-time modelling of wavepackets in turbulent jets. Journal of Fluid Mechanics 821, 458-481.

Savarese, A, Jordan, P, Girard, S, Royer, A, Fourment, C, Collin, E, Gervais, Y \& PORTA, M 2013 Experimental study of shock-cell noise in underexpanded supersonic jets. AIAA Paper 2080, 2013.

Sinha, A, Rodríguez, D, BrÈs, GA \& Colonius, T 2014 Wavepacket models for supersonic jet noise. Journal of Fluid Mechanics 742, 71-95.

SuZuKI, T 2013 Coherent noise sources of a subsonic round jet investigated using hydrodynamic and acoustic phased-microphone arrays. Journal of Fluid Mechanics 730, 659.

SuZUKI, T 2016 Wave-packet representation of shock-cell noise for a single round jet. AIAA Journal . 
Suzuki, T \& Colonius, T 2006 Instability waves in a subsonic round jet detected using a near-field phased microphone array. Journal of Fluid Mechanics 565, 197-226.

TAM, CKW 1987 Stochastic model theory of broadband shock associated noise from supersonic jets. Journal of Sound and Vibration 116 (2), 265-302.

TAM, CKW 1990 Broadband shock-associated noise of moderately imperfectly expanded supersonic jets. Journal of Sound and Vibration 140 (1), 55-71.

TAM, CKW 1995 Supersonic jet noise. Annual Review of Fluid Mechanics 27 (1), 17-43.

TAM, CKW, JACKSOn, JA \& SEINER, JM 1985 A multiple-scales model of the shock-cell structure of imperfectly expanded supersonic jets. Journal of Fluid Mechanics 153, 123149.

TAM, CKW, SeIner, JM \& YU, JC 1986a Proposed relationship between broadband shock associated noise and screech tones. Journal of sound and vibration 110 (2), 309-321.

TAm, CKW, Seiner, JM \& Yu, JC 1986b Proposed relationship between broadband shock associated noise and screech tones. Journal of sound and vibration 110 (2), 309-321.

TAM, CKW \& TANnA, HK 1982 Shock associated noise of supersonic jets from convergentdivergent nozzles. Journal of Sound and Vibration 81 (3), 337-358.

Tan, DJ, Kalyan, A, Gryazev, V, Wong, M, Honnery, D, Edgington-Mitchell, DM \& KARABASOV, SA 2017 On the application of shock-associated noise models to piv measurements of screeching axisymmetric cold jets. In 23rd AIAA/CEAS Aeroacoustics Conference, p. 3028.

Towne, A, Colonius, T, Jordan, P, Cavalieri, AVG \& Bres, GA 2015 Stochastic and nonlinear forcing of wavepackets in a mach 0.9 jet. AIAA paper 2217, 2015.

Troutt, TR \& McLaughlin, DK 1982 Experiments on the flow and acoustic properties of a moderate-reynolds-number supersonic jet. Journal of Fluid Mechanics 116, 123-156.

Williams, JE Ffowcs \& Kempton, AJ 1978 The noise from the large-scale structure of a jet. Journal of Fluid Mechanics 84 (4), 673-694.

Zhang, MQ, Jordan, P, Lehnasch, G, Cavalieri, AVG \& Agarwal, A 2014 Just enough jitter for jet noise? In 20th AIAA/CEAS Aeroacoustics Conference, p. 3061. 